\author{
Universidade de São Paulo \\ Instituto de Física \\ Departamento de Física Matemática
}

\title{
Largura do Bóson de Higgs em Dois Fótons em Modelos além do Modelo Padrão
}

\author{
Rômulo Brandão Madeira
}

Orientadora: Profa. Dra. Renata Zukanovich Funchal São Paulo

2016 



\author{
Universidade de São Paulo \\ Instituto de Física \\ Departamento de Física Matemática
}

\title{
Largura do Bóson de Higgs em Dois Fótons em Modelos além do Modelo Padrão
}

\author{
Rômulo Brandão Madeira
}

ORIENTADORA: Profa. Dra. Renata Zukanovich Funchal

Dissertação apresentada ao Programa de Pós-Graduação em

Física do Instituto de Física de São Paulo da Universidade de

São Paulo, para obtenção do título de Mestre em Ciências.

\section{BANCA EXAMINADORA:}

Profa. Dra. Renata Zukanovich Funchal (IFUSP)

Prof. Dr. Edivaldo Moura Santos (IFUSP)

Prof. Dr. André Paniago Lessa (UFABC)

São Paulo

2016 


\section{FICHA CATALOGRÁFICA \\ Preparada pelo Serviço de Biblioteca e Informação do Instituto de Física da Universidade de São Paulo}

Madeira, Rômulo Brandão

Largura do bóson de Higgs em dois fótons em modelos além do modelo padrão. São Paulo, 2016.

Dissertação (Mestrado) - Universidade de São Paulo. Instituto de Física. Depto. de Física Matemática

Orientador: Profa. Dra. Renata Zukanovich Funchal

Área de Concentração: Física das Partículas Elementares e Campos.

Unitermos: 1. Física; 2. Física de alta energia; 3. Física de partículas; 4. Partículas elementares; 5. LHC. 


\section{Agradecimentos}

Agradeço a todas as pessoas e instituições que me prestaram apoio para finalizar este trabalho. Em especial, agradeço aos meus pais, Edna Régia Brandão Madeira e Raimundo Neto Madeira, e aos meus irmãos, Eveline Brandão Madeira e Ramon Brandão Madeira, por me motivarem a seguir o mestrado até o fim. Sem eles tudo seria mais difícil.

Agradeço à minha orientadora Renata Zukanovich Funchal por ter servido a mim como exemplo de competência e seriedade no trabalho. Também, agradeço pela paciência e por entender os problemas pelos quais estamos sujeitos a passar.

Não poderia esquecer dos familiares que estiveram juntos a mim durante o período que estive em São Paulo: meus primos Nayron e Maihara e meus tios Nicinha e Durval. Sou bastante grato a tudo que eles fizeram por mim.

Agradeço aos amigos Antonio Sales e Jorgivan Dias que foram importantes na minha adaptação à cidade de São Paulo, ao Yuber Ferney que foi a pessoa que mais me ajudou tirando dúvidas sobre a minha dissertação e aos amigos Túlio Brito, Cristian Caniu, César Nieto, Yadira Patria e Walace Elias que me serviram de companhia durante os anos do mestrado.

Também, agradeço à USP, CAPES e CNPQ, pelo suporte financeiro e de infra-estrutura. 



\section{Resumo}

Nesta dissertação, revisamos o desenvolvimento do mecanismo de Higgs e a sua importância para a estruturação do Modelo Padrão da física de partículas elementares sob um ponto de vista histórico e contemporâneo. Foi enfatizado como a introdução de um dubleto escalar complexo com as simetrias de gauge do modelo fez com que algumas partículas adquirissem massa. Foi apresentado o contexto da descoberta do bóson de Higgs consumada em 2012 e possíveis discrepâncias nos resultados obtidos no Large Hadron Collider que poderiam indicar a possibilidade de física nova, em especial no canal de decaimento $H \rightarrow \gamma \gamma$. Estudamos as contribuições para a largura deste bóson em dois fótons com a introdução de novas partículas fermiônicas e escalares. Uma síntese do cálculo da largura deste canal, assim como as técnicas envolvidas para realizá-lo, são apresentadas. Considerando apenas as duas contribuições dominantes, foi estimado um valor de 9,165 $\mathrm{keV}$ para a largura do decaimento, resultando em uma razão de ramificação de $2,24 \cdot 10^{-3}$.

Palavras-chave: Decaimento $H \rightarrow \gamma \gamma$, Bóson de Higgs, Mecanismo de Higgs, Teorias de Gauge, Modelo Padrão, LHC. 



\section{Abstract}

In this dissertation, we review the development of the Higgs mechanism and its importance to the structure of the Standard Model of elementary particles physics from a historical and contemporary point of view. It was emphasized how the introduction of a complex scalar doublet with the gauge symmetries of the model induces some particles to acquire mass. It was presented the context of Higgs boson discovery accomplished in 2012 and possible discrepancies in the results obtained at the Large Hadron Collider which may indicate the possibility of new physics, in particular in the $H \rightarrow \gamma \gamma$ decay channel. We studied the contributions to the width of this boson decay into two photons with the introduction of new fermionic and scalar particles. A synthesis of computation of this channel width, as well as techniques involved to accomplish it, are presented. Considering the only two dominant contributions, it was estimated a value of $9.165 \mathrm{keV}$ for the decay width, resulting in a branching ratio of $2.24 \cdot 10^{-3}$.

Keywords: $H \rightarrow \gamma \gamma$ Decay, Higgs Boson, Higgs Mechanism, Gauge Theories, Standard Model, LHC. 



\section{Sumário}

$\begin{array}{ll}\text { Introdução } & 13\end{array}$

O desenvolvimento do mecanismo de Higgs . . . . . . . . . . . . . 14

A descoberta do bóson de Higgs no detector ATLAS . . . . . . . 19

A descoberta do bóson de Higgs no detector CMS . . . . . . . . . . 22

Resultados atualizados para o bóson de Higgs . . . . . . . . . . . . 27

Novas pesquisas para o bóson de Higgs a partir do Run-2 do LHC . 29

A proposta desta dissertação . . . . . . . . . . . . . . . . . . . . . . . . 29

1 Introdução ao modelo padrão eletrofraco 30

1.1 O setor de gauge no modelo padrão eletrofraco . . . . . . . . . 32

1.2 O mecanismo de Higgs no modelo padrão eletrofraco . . . . . 34

1.3 Considerações sobre o setor fermiônico no modelo padrão ele-

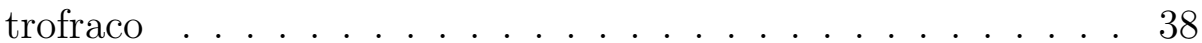

2 A largura de decaimento $H \rightarrow \gamma \gamma$ e as diversas contribuições possíveis

2.1 A contribuição dos loops de férmions no decaimento $H \rightarrow \gamma \gamma$. 45

2.2 A contribuição dos loops dos bósons de gauge $\mathrm{W}$ no decaimento $H \rightarrow \gamma \gamma \ldots \ldots \ldots$. . . . . . . . . . 47

2.3 A contribuição dos loops de partículas escalares no decaimento $H \rightarrow \gamma \gamma \ldots \ldots \ldots \ldots \ldots \ldots$

2.4 A largura do bóson de Higgs no decaimento $H \rightarrow \gamma \gamma \ldots$. . . . 51

Conclusões 
A Técnicas para cálculos de integrais de loop 58

A.1 Propriedades do traço das matrizes $\gamma$. . . . . . . . . . . 58

A.2 Parâmetros de Feynman . . . . . . . . . . . . . . . . . 59

A.3 Regularização dimensional . . . . . . . . . . . . . . . . 60 


\section{Lista de Figuras}

1 Intensidade do sinal $\mu=\frac{\sigma}{\sigma_{M P}}$ para $m_{H}=126 \mathrm{GeV}$. O valor para os resultados combinados é $\mu=1,4 \pm 0,3$ obtido no detector ATLAS [11]. . . . . . . . . . . . . . . . . . . 21

2 Relação entre o sinal $\mu$ e a massa $m_{H}$ representada pelos intervalos de confiança para os canais $H \rightarrow W W \rightarrow l \nu l \nu, \gamma \gamma$ e $Z Z[11] \ldots \ldots \ldots \ldots \ldots \ldots \ldots$

3 Intensidade do sinal definido como $\frac{\sigma}{\sigma_{M P}}$ em função da massa do bóson de Higgs para os dados combinados de medidas a energias de centro-de-massa de $7 \mathrm{TeV}$ e $8 \mathrm{TeV}$. A faixa verde corresponde à região de erro compreendida entre $+\sigma$ e $-\sigma$ (desvio-padrão) das medidas [12] . . . . . . . . . . . . 25

4 Intensidade do sinal $\frac{\sigma}{\sigma_{M P}}$ para cinco canais de decaimento medidos (pontos pretos) e para suas combinações (linha vertical preta). As barras horizontais vermelhas representam as regiões de $\pm \sigma$ em torno da média obtida para cada canal; a barra verde representa a região de $\pm 0,23$ em torno da média 0,87 dos canais combinados [12]. . . . . . . . . . . . . 26

5 Resultados atualizados para a intensidade do sinal de diversos canais (ATLAS+CMS) [45]. . . . . . . . . . . . . 27

6 Ajuste dos resultados atualizados para a intensidade do sinal de diversos canais (ATLAS+CMS) e suas respectivas barras de erro $( \pm 1 \sigma)$ e $( \pm 2 \sigma)[45] \ldots \ldots . \ldots . \ldots 28$ 
1.1 O potencial do campo $\Phi$ na situação de quebra espontânea de simetria. As setas indicam como os campos $\vec{\xi}(x)$ de Goldstone e $H(x)$ de Higgs parametrizam as excitações em torno de um estado de mínima energia [48]. . . . . . . . . . . . . . 36

2.1 Função de loop para contribuição das partículas de spin $\frac{1}{2}$, equação (2.28). . . . . . . . . . . . . . . . . . . 52

2.2 Função de loop para contribuição das partículas de spin 1, equação (2.29). . . . . . . . . . . . . . . . . 53

2.3 Função de loop para contribuição das partículas de spin 0, equação (2.30). . . . . . . . . . . . . . . . 54 


\section{Lista de Tabelas}

1.1 Atual estrutura da matéria conhecida. . . . . . . . . . . . 32 


\section{Introdução}

Durante os últimos quarenta anos, o Modelo Padrão (MP) da física de partículas elementares $[1,2,3,4]$ foi submetido a diversos testes experimentais. Os resultados mostraram que este modelo é bem sucedido na descrição das interações entre partículas a altas energias.

Nos anos 1960, as primeiras tentativas de descrever as interações entre léptons e entre léptons e fótons surgiram sob a hipótese de que elas eram intermediadas por bósons vetoriais [1]. Aparentemente, até então, nenhuma relação existia entre os efeitos eletromagnéticos e os provenientes da interação fraca. A motivação por ter em mãos uma teoria matemática elegante e consistente com os dados experimentais movimentou a comunidade científica de física de partículas durante todos esses anos. No começo, no entanto, essas tentativas foram frustradas.

Em teorias de gauge, as interações aparecem naturalmente pela introdução de campos mediadores vetoriais necessários para manter a simetria, assim como é feito na eletrodinâmica quântica. Nas interações fracas, surge um outro problema. Elas são de curto alcance, portanto as partículas mediadoras devem ser massivas. No entanto, a conservação da simetria de gauge impede que tais partículas possuam massa. Este problema é resolvido a partir de um mecanismo que permite a existência de bósons massivos e que respeitam a simetria de gauge.

O MP trata léptons e quarks como constituintes básicos da matéria. As interações entre eles se dão por meio da troca de partículas mediadoras das interações. Para as interações eletromagnéticas há o fóton, para as interações 
fracas há os bósons W e Z e para as interações fortes há os glúons. As duas primeiras interações foram unificadas em uma teoria chamada eletrofraca. O MP descreve essas interações de forma precisa e de acordo com muitos resultados obtidos nos experimentos realizados em aceleradores de partículas.

O MP introduz um dubleto escalar que adquire um valor esperado de vácuo não nulo e, assim, fazendo uso de um mecanismo proposto há cinquenta anos $[5,6,7,8,9,10]$ é capaz de explicar por que os bósons $\mathrm{W}$ e $\mathrm{Z}$ possuem massa enquanto o fóton continua com massa nula. Mais detalhes de como este processo se aplica no contexto do Modelo Padrão Eletrofraco (MPE) será apresentado no Capítulo 1.

O mecanismo descrito é conhecido como mecanismo de Higgs. Além de ele dar massa às partículas $\mathrm{W}$ e Z, também dá aos férmions fundamentais e prevê a existência de uma partícula nova conhecida como bóson de Higgs. Sua massa $\left(m_{H}\right)$ não é prevista por este modelo, pois trata-se de uma entidade dependente de um parâmetro livre da teoria. A nova partícula surge ao introduzir um dubleto escalar complexo proposto à teoria eletrofraca $[1,2,3]$.

\section{O desenvolvimento do mecanismo de Higgs}

O mecanismo de Higgs não havia sido comprovado experimentalmente até 2012, quando foi anunciada a descoberta no acelerador Large Hadron Collider (LHC) de uma partícula que obedece às características do bóson de Higgs $[11,12]$. Esta partícula prevista teoricamente era a única partícula não observada do MP até então. Um dos principais objetivos do LHC era identificar ou excluir a existência deste bóson [13].

Teorias cujas lagrangeanas são simétricas mas não o seu estado de vácuo foram bastante importantes no entendimento de fenômenos da supercondutividade. Vários autores sugeriram que teorias semelhantes pudessem ser empregadas também na física de partículas $[14,15,16,17]$. Porém, partículas indesejadas sem massa que surgem nessas teorias foram entraves para o de- 
senvolvimento de uma teoria que abrangesse fenômenos de altas energias.

Problemas de teorias com quebra de simetria para física de partículas foram apresentados por Nambu, Jona-Lasinio e Pascual no começo dos anos 1960 [14, 15, 18] e complementados por Baker e Glashow [16] e Glashow [17] em 1962. Os trabalhos de Goldstone [19] (1961), Goldstone, Salam e Weinberg [20] (1962) e Bludman e Klein [21] (1963) também exibiram estudos sobre a mesma questão, onde também mostraram que em tais teorias ocorre a existência de bósons vetoriais sem massa que restauram a simetria.

Estes bósons indesejados são conhecidos como bósons de Goldstone e, desde o início dos estudos de teorias com quebra de simetria em física de partículas, procurou-se uma forma de evitá-los. Esses trabalhos deram suporte para o desenvolvimento do mecanismo que serviu de peça para a construção do MP.

Dentre os diversos trabalhos que trataram de apresentar formas de eliminar esses bósons sem massa, Higgs mostrou que a forma de unir teorias com quebra de simetria e ausência de partículas sem massa é acoplando-as com campos de gauge [9]. Já Kibble introduziu uma generalização dos modelos apresentados até então para um grupo não-Abeliano arbitrário [10].

Foi em 1964, que Englert e Brout mostraram que, em certos casos, mésons vetoriais adquirem massa quando o vácuo é degenerado com respeito a um grupo de Lie [5]. Foram estudados dois casos: um com uma partícula bosônica $\phi$ carregada interagindo com um campo de gauge $A_{\mu}$ (depois generalizada para várias destas partículas); e um com partículas fermiônicas $\psi$ interagindo com campos de gauge vetorial $A_{\mu}$ e pseudovetorial $B_{\mu}$.

Higgs propôs que, em algumas teorias de campos covariantes de Lorentz, acoplamentos com campos de gauge de spin 1 proporcionam o surgimento de massa [7]. Foram estudados os casos analisados por Goldstone [19] e Goldstone, Salam e Weinberg [20] onde três campos $\phi_{1}, \phi_{2}$ (escalares) e $A_{\mu}$ (vetorial) se interagem de acordo com a Lagrangeana:

$$
L=-\frac{1}{2}\left(\nabla \phi_{1}\right)^{2}-\frac{1}{2}\left(\nabla \phi_{2}\right)^{2}-V\left(\phi_{1}^{2}+\phi_{2}^{2}\right)-\frac{1}{4} F_{\mu \nu} F^{\mu \nu}
$$


onde ( $e$ sendo uma constante de acoplamento)

$$
\begin{aligned}
\nabla_{\mu} \phi_{1} & =\partial_{\mu} \phi_{1}-e A_{\mu} \phi_{2}, \\
\nabla_{\mu} \phi_{2} & =\partial_{\mu} \phi_{2}+e A_{\mu} \phi_{1} \mathrm{e} \\
F_{\mu \nu} & =\partial_{\mu} A_{\nu}-\partial_{\nu} A_{\mu}
\end{aligned}
$$

e $V$ refere-se ao termo pontencial cuja simetria pode ser quebrada no seu estado de vácuo.

Na situação de quebra espontânea da simetria, escolhe-se a solução para o vácuo $\phi_{1}(x)=0, \phi_{2}(x)=\phi_{0}$. Tratando $\Delta \phi_{1}$ e $\Delta \phi_{2}$ como pequenas variações em torno desta solução e descrevendo (1) em termo destes campos, chegamos ao espectro de partículas descrito pelas seguintes equações:

$$
\begin{aligned}
\left\{\partial^{2}-4 \phi_{0}^{2} V^{\prime \prime}\left(\phi_{0}^{2}\right)\right\}\left(\Delta \phi_{2}\right) & =0 \\
\partial_{\mu} B^{\mu}=0, \partial_{\nu} G^{\mu \nu}+e^{2} \phi_{0}^{2} B^{\mu} & =0
\end{aligned}
$$

onde os campos $B_{\mu}$ e $G_{\mu \nu}$ são definidos como:

$$
\begin{aligned}
B_{\mu} & =A_{\mu}-\left(e \phi_{0}\right)^{-1} \partial_{\mu}\left(\Delta \phi_{1}\right), \\
G_{\mu \nu} & =\partial_{\mu} B_{\nu}-\partial_{\nu} B_{\mu} .
\end{aligned}
$$

As equações (5) e (6) representam um campo escalar de massa $2 \phi_{0}\left\{V^{\prime \prime}\left(\phi_{0}^{2}\right)\right\}^{1 / 2}$ e um vetorial de massa $e \phi_{0}$, respectivamente. O surgimento dessas massas ocorre por meio do acoplamento de $A_{\mu}$ com os campos $\phi_{1}$ e $\phi_{2}$, já que para $e=0$ o fenômeno não mais acontece.

No trabalho de Guralnik, Hagen e Kibble de 1964 [8], também foi notado que é possível desenvolver uma teoria de campos simples cuja quebra da simetria não necessariamente excita partículas de massa zero, como afirmaram Goldstone [19] e Goldstone, Salam e Weinberg [20]. Este trabalho serviu como procedimento geral para eliminação de bósons sem massa indesejados.

Como exemplo, foi analisado o caso da eletrodinâmica de partículas de spin zero, inicialmente sem massa, estudada por Englert e Brout [5]. A lagrangeana que a retrata é:

$$
L=-\frac{1}{2} F^{\mu \nu}\left(\partial_{\mu} A_{\nu}-\partial_{\nu} A_{\mu}\right)+\frac{1}{4} F^{\mu \nu} F_{\mu \nu}+\phi^{\mu} \partial_{\mu} \phi+\frac{1}{2} \phi^{\mu} \phi_{\mu}+i e_{0} \phi^{\mu} \sigma_{2} \phi A_{\mu},
$$


onde $\phi$ representa um campo escalar de duas componentes $\phi_{1}$ e $\phi_{2}$; e $\phi^{\mu}=$ $\left(\partial^{\mu}-i e A^{\mu}\right) \phi$.

Ao impor a condição de quebra espontânea da simetria na lagrangeana (9) e escolhendo uma solução para o vácuo, obtemos as seguintes equações de movimento:

$$
\begin{aligned}
\left(-\partial^{2}+\eta_{1}^{2}\right) \phi_{1} & =0 \\
\left(-\partial^{2}+\eta_{1}^{2}\right) A_{k}^{T} & =0 \\
-\partial^{2} \phi_{2}^{2} & =0
\end{aligned}
$$

onde o elemento $\eta_{1}$ surge nas condições de quebra da simetria e o índice $T$ indica "parte transversa". Observa-se que as duas primeiras equações combinadas formam um campo vetorial com massa $\eta_{1}$ e a terceira equação representa um campo escalar sem massa.

Até a época do desenvolvimento do mecanismo de Higgs, o único bóson vetorial sem massa conhecido era o fóton. Na teoria eletrofraca, os bósons de gauge intemediários deveriam possuir massas muito superiores aos hádrons conhecidos. Contudo, Englert e Brout [5] e Higgs [7] mostraram que após a quebra espontânea da simetria em determinada teoria com acoplamentos fracos, mésons vetoriais adquirem massa.

Fenômenos estudados classicamente mostraram que a polarização longitudinal de uma excitação vetorial massiva substitui uma excitação escalar sem massa [7]. Higgs usou um modelo com um grupo de simetria $U(1)$ para incorporar os estudos já realizados sobre a quebra espontânea de simetria até então [9]. Foi considerado uma dupla de campos escalares $\phi_{1}(x)$ e $\phi_{2}(x)$ e um campo de gauge vetorial $A_{\mu}(x)$.

A lagrangeana do modelo estudado em [9] é a mesma dada pela equação (1) e pelas condições (2), (3) e (4), onde o potencial $V\left(\phi_{1}^{2}+\phi_{2}^{2}\right)$ é dado como $\frac{1}{8} f^{2}\left(\phi_{a} \phi_{a}\right)^{2}-\frac{1}{4} m_{0}^{2} \phi_{a} \phi_{a}$. Apesar do último termo parecer um elemento de massa, ele não o é, pois o sinal está propositalmente errado.

A teoria descrita acima admite uma série de soluções para o equilíbrio:

$$
\phi_{1}=\eta \cos \alpha, \phi_{2}=\eta \sin \alpha,
$$


onde $\eta=\frac{m_{0}}{f}$ e $\alpha$ é um parâmetro variável cujo valor define as soluções. Escolhendo $\alpha=\pi / 2$ e tratando os campos $A_{\mu}, \phi_{1}$ e $\phi_{2}-\eta$ como valores pequenos, chega-se às seguintes equações de movimento:

$$
\begin{gathered}
\partial_{\mu} F^{\mu \nu}=-e^{2} \eta^{2} B^{\mu} \quad, \partial_{\mu} B^{\mu}=0 \\
\left(\square-m_{0}^{2}\right) \chi=0,
\end{gathered}
$$

onde foram usadas as seguintes substituições:

$$
\begin{gathered}
B_{\mu}=A_{\mu}-(e \eta)^{-1} \partial_{\mu} \phi \\
\phi=\phi_{1}, \quad \chi=\phi_{2}-\eta .
\end{gathered}
$$

Após a quantização dos campos dados, as equações (14) e (15) representam dois bósons livres, um vetorial com massa e $\eta$ e um escalar com massa $m_{0}$.

Em resumo, o que foi observado é que os mecanismos desenvolvidos permitiram que campos sem massa pudessem ser transformados em estados longitudinais de bósons vetoriais massivos. A combinação do mecanismo da quebra espontânea da simetria com as transformações de gauge serviu de base para o desenvolvimento do MP para física de partículas. Simetrias do tipo $S U(3)$ são tratadas de forma semelhante.

No trabalho de Kibble [10], considerou-se um campo escalar real $\phi \operatorname{com} n$ componentes que se transformam como uma representação de um grupo de Lie de dimensão $g$ :

$$
\phi(x) \rightarrow e^{\lambda \cdot T} \phi(x)
$$

onde

$$
\lambda \cdot T=\lambda^{A} T_{A},
$$

$\lambda^{A}$ são $g$ parâmetros reais e $T_{A}$ são as $g$ matrizes que obedecem às relações de comutação do grupo de Lie em consideração. A lagrangeana é:

$$
L=\phi^{\mu} \partial_{\mu} \phi-\frac{1}{2} \phi^{\mu} \phi_{\mu}-V(\phi)
$$

onde $\phi^{\mu}=\left(\partial^{\mu}-i e A^{\mu}\right) \phi$ e $V(\phi)$ é invariante sob a rotação (18).

Sendo $\langle\phi\rangle=\eta$ uma solução para o valor esperado de vácuo com simetria quebrada, supomos que apenas $\nu$ componentes são diferentes de zero na forma 
canônica de $\eta$ e $r$ componentes são iguais a zero: $n=r+\nu$. Observa-se que a teoria possui $n-r=\nu$ campos escalares massivos e que, após aplicar o mecanismo da quebra de simetria, as $r$ componentes restantes dos campos escalares combinam-se com $r$ campos vetoriais formando $r$ campos vetoriais massivos. Portanto, nos restam $g-r$ campos vetoriais sem massa.

Para evitar que na teoria surjam campos sem massa é necessário que escolhamos $r=g$. Nos casos onde a teoria obedece às simetrias de gauge não-abelianas, a introdução de campos de gauge acoplados aos campos escalares elimina partículas sem massa. Assim, cada componente com simetria não-quebrada mantém um campo vetorial sem massa. Apesar da aparente facilidade em arbitrar teorias usando este resultado, dificuldades surgem nos acoplamentos; pois os valores para as massas previstas nas teorias devem estar de acordo com o observado nos experimentos.

\section{A descoberta do bóson de Higgs no detector ATLAS}

A Toroidal LHC Apparatus (ATLAS) é um dos detectores multipropósitos para física de partículas que compõe o LHC [22, 23, 24] e possui geometria cilíndrica. Um conjunto de sistemas combinados neste detector fornece medidas precisas de léptons, fótons e partículas carregadas.

Os resultados apresentados nesta seção são referentes aos resultados parciais do run-1 do LHC publicados em Agosto de 2012. Dados combinados do detector ATLAS recentes serão apresentados adiante e fornecem medidas mais precisas para as seções de choque.

Alguns limites indiretos calculados estabeleceram uma massa para o bóson de Higgs menor que $158 \mathrm{GeV}$ [25]. Em 2011, medidas obtidas na colisão próton-próton $(p p)$ com energia de centro de massa de $\sqrt{s}=7 \mathrm{TeV}$ no detector ATLAS do LHC revelaram um excesso de eventos numa região de massa entre 124 e $126 \mathrm{GeV}$. Entre Abril e Junho de 2012, novas medidas 
foram obtidas no mesmo detector [11], agora a uma energia de centro de massa de $\sqrt{s}=8 \mathrm{TeV}$ na colisão $p p$. As buscas foram feitas nos canais $H \rightarrow Z Z^{(*)} \rightarrow 4 l^{1}, H \rightarrow \gamma \gamma, H \rightarrow W W^{(*)} \rightarrow e \nu \mu \nu$.

Medidas já haviam encontrado um limite $m_{H}<152 \mathrm{GeV}$ [26] e considerações teóricas sugeriram um limite máximo de $1 \mathrm{TeV}$ para $m_{H}[27,28$, 29, 30]. Buscas pelo bóson de Higgs realizadas durante os últimos vinte anos no colisor LEP encontraram um limite $m_{H}>114,4 \mathrm{GeV}$ [31]. No mesmo período, buscas no colisor próton-antipróton do Tevatron excluíram a região de massa que varia de 162 a $166 \mathrm{GeV}$ [32] para $m_{H}$ e também detectou um excesso de eventos na região de 120 a $135 \mathrm{GeV}[33,34,35]$.

No canal de decaimento $H \rightarrow Z Z^{(*)} \rightarrow 4 l$, a busca pelo bóson de Higgs do MP fornece uma boa determinação para massas entre 110 e $600 \mathrm{GeV}$ devido, principalmente, à excelente resolução do detector ATLAS em medidas de momentos. Os dados para energia de centro de massa de $\sqrt{s}=7 \mathrm{TeV}$ são confrontados com os dados para energia de $\sqrt{s}=8 \mathrm{TeV}$ [11]. A análise seleciona dois pares de léptons isolados para a busca pelo bóson de Higgs. Cada um dos pares é formado por dois léptons de cargas opostas e mesmo sabor.

No decaimento $H \rightarrow \gamma \gamma$, a busca pelo bóson de Higgs do MP foi realizada na região de massa que varia de 110 a $150 \mathrm{GeV}$. Os dados obtidos a uma energia de centro de massa de $\sqrt{s}=7 \mathrm{TeV}$ [36] foram comparados com os obtidos em 2012 a uma energia de $\sqrt{s}=8 \mathrm{TeV}$ cuja análise teve sensibilidade melhorada em relação à anterior [11].

A busca pelo bóson de Higgs no canal $H \rightarrow W W^{(*)} \rightarrow e \nu \mu \nu$ a uma energia de $8 \mathrm{TeV}$ foi realizada na região de massas que varia de 110 a $200 \mathrm{GeV}$ [11]. A análise dos dados foram comparadas com as obtidas a uma energia de 7 $\mathrm{TeV}$ descritas em [37].

Os dados obtidos para os canais $H \rightarrow Z Z^{(*)} \rightarrow 4 l, H \rightarrow \gamma \gamma$ e $H \rightarrow$ $W W^{(*)} \rightarrow e \nu \mu \nu$ a uma energia de centro de massa de $\sqrt{s}=8 \mathrm{TeV}$ e também a reanálise dos dados a uma energia de $\sqrt{s}=7 \mathrm{TeV}$ para os dois primeiros

\footnotetext{
${ }^{1} l$ significa elétron ou múon
} 
canais possibilitaram um ganho de sensibilidade na medida das massas se comparado com o obtido em [38].

No experimento do ATLAS de 2012, foi observado uma exclusão para a massa do bóson de Higgs do MP em uma faixa que varia de 111 a $559 \mathrm{GeV}$, exceto a região que varia de 122 a $131 \mathrm{GeV}$, onde observou-se um excesso de eventos. Os resultados evidenciam a existência de uma partícula nova com massa próxima de $126 \mathrm{GeV}[11]$.

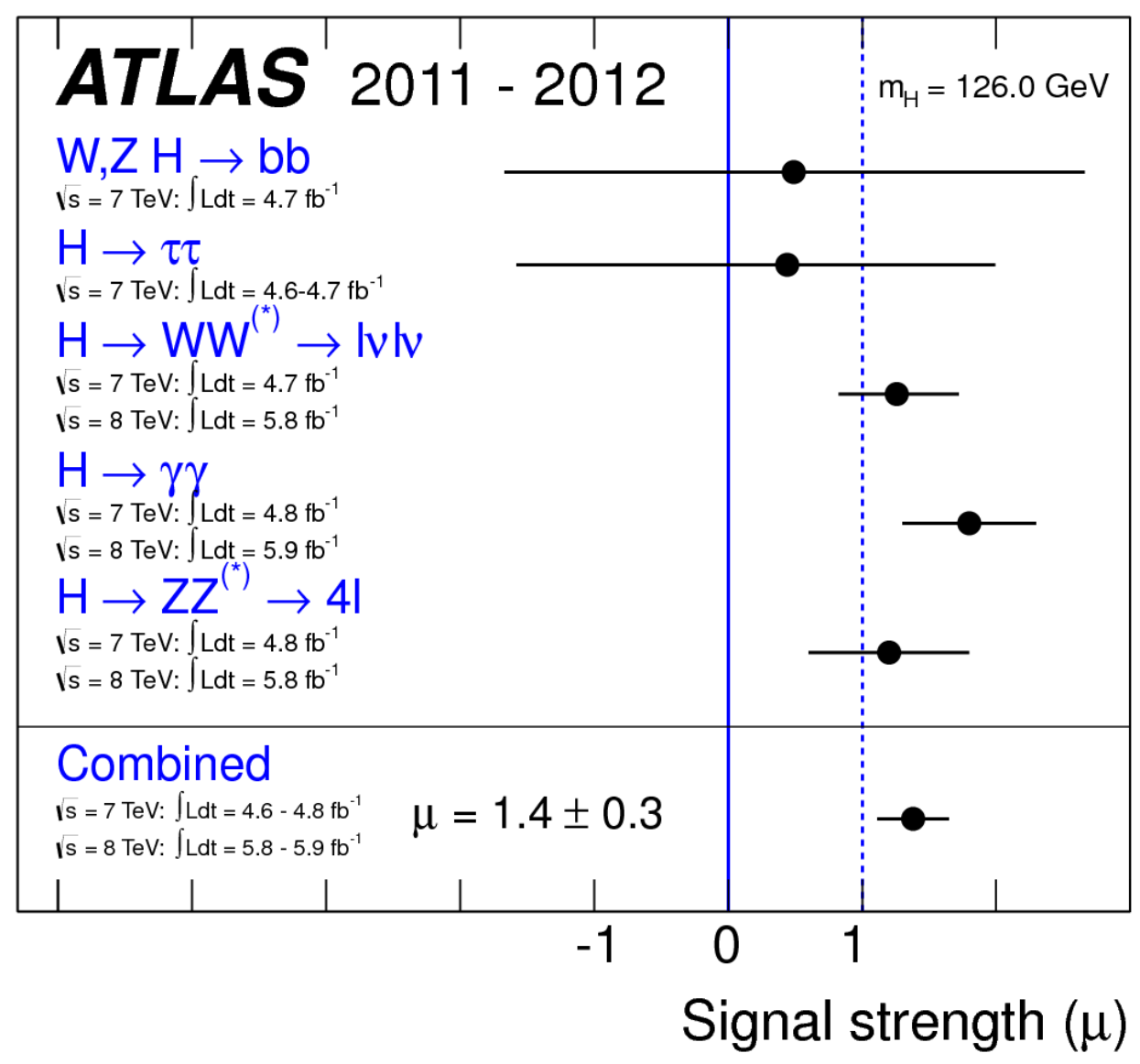

Figura 1: Intensidade do sinal $\mu=\frac{\sigma}{\sigma_{M P}}$ para $m_{H}=126 \mathrm{GeV}$. O valor para os resultados combinados é $\mu=1,4 \pm 0,3$ obtido no detector ATLAS [11].

A partícula nova observada pode ser reconhecida como um bóson neutro. 
A identificação do decaimento dela em dois bósons vetoriais cuja soma das cargas elétricas é zero evidencia isto. Na busca realizada no ATLAS [11], concluiu-se que a partícula preenche as lacunas experimentais referentes ao bóson de Higgs previsto pelo MP, porém ainda mais medidas seriam necessárias para aprofundar na sua natureza.

A Figura 1 resume os valores do sinal $\mu=\frac{\sigma}{\sigma_{M P}}$ para os canais de decaimentos individualmente e combinados para $m_{H}=126 \mathrm{GeV}$. A intensidade do sinal $\mu$ representa a razão entre seção de choque medida e a seção de choque hipotética do MP. Estes valores são referentes aos primeiros resultados obtidos de 2012, portanto não tratam-se das medidas mais atualizadas. O excesso de eventos para esse valor de $m_{H}$ corresponde a um valor para o sinal de $\mu=1,4 \pm 0,3$ [11]. Observa-se que os canais $H \rightarrow W W, \gamma \gamma$ e $Z Z$ possuem menores barras de erros em suas medidas, justificadas por serem os canais de melhor resolução.

Observa-se também, que os resultados obtidos em 2011/2012 no detector ATLAS apontavam para uma razão de ramificação para o decaimento $H \rightarrow$ $\gamma \gamma$ de $\mu=1,8 \pm 0,5$, quase duas vezes maior que o esperado teoricamente. Isto pôde sugerir que a largura parcial do Higgs em dois fótons calculada poderia estar incompleta e que modificações nela poderiam ser sinal de física nova.

Regiões para níveis de confiança de $68 \%$ e $95 \%$ do sinal $\mu$ em função da massa do bóson de Higgs são apresentadas na Figura 2. Este resultado também não é o mais atualizado e é referente às primeiras medidas apresentadas em 2012. Os canais analisados correspondem a $H \rightarrow W W \rightarrow l \nu l \nu, \gamma \gamma$ e $Z Z$ para dados combinados a energias de $\sqrt{s}=7 \mathrm{TeV}$ e $8 \mathrm{TeV}$ [11].

\section{A descoberta do bóson de Higgs no detector CMS}

Em 2011, buscas diretas pelo bóson de Higgs foram feitas também no 


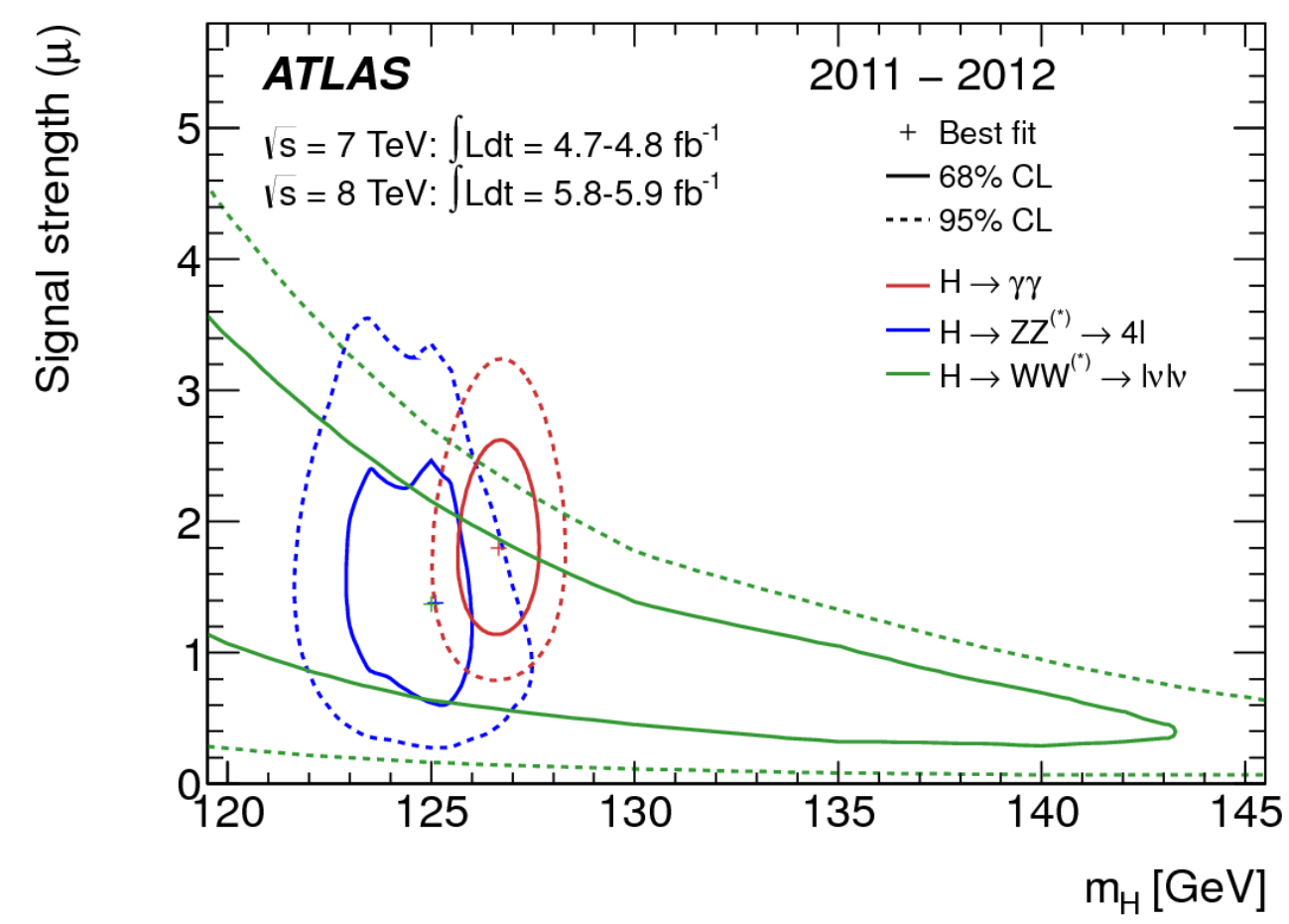

Figura 2: Relação entre o sinal $\mu$ e a massa $m_{H}$ representada pelos intervalos de confiança para os canais $H \rightarrow W W \rightarrow l \nu l \nu, \gamma \gamma$ e $Z Z$ [11]

detector Compact Muon Solenoid (CMS) do LHC a uma energia de centro de massa de $\sqrt{s}=7 \mathrm{TeV}$ em colisão pp. Neste detector, a região de 127 a $600 \mathrm{GeV}$ foi excluída do espectro de massas [39].

Os detectores CMS e ATLAS foram construídos com uma finalidade de analisar todos os canais de decaimento aptos a identificar o bóson de Higgs sobre uma grande faixa de massas e, além disso, capaz de identificar qualquer sinal de física nova. Como o valor de $m_{H}$ não é previsto pela teoria e o valor da largura do bóson de Higgs varia bastante em função do valor da sua massa, então as buscas pelo bóson foram realizadas sobre uma grande quantidade de possíveis $m_{H}$ e sobre diversos canais de decaimento.

O projeto do experimento do CMS foi embasado na possibilidade de indentificar o bóson de Higgs do MP, que serviu como referência para avaliar o funcionamento do detector $[40,41,42]$. O aparato é formado por um so- 
lenóide supercondutor, que fornece um campo magnético de 3, $8 \mathrm{~T}$, de $6 \mathrm{~m}$ de diâmetro interno [43]. O software do CMS executa numerosas simulações de eventos no detector e também a reconstrução e análise dos dados coletados nas medidas.

No experimento de 2012 do detector CMS do LHC, a energia de centro de massa de uma colisão pp foi aumentada para $\sqrt{s}=8 \mathrm{TeV}$ e, também, houve um aumento na sensibilidade das medidas. As buscas realizadas no CMS foram feitas nos modos de decaimento mais sensíveis na região de baixos valores para massa: $H \rightarrow \gamma \gamma, Z Z, W^{+} W^{-}, \tau^{+} \tau^{-}$e $b \bar{b}$ na região de massa que varia de 110 a $160 \mathrm{GeV}$. Daqui em diante, $H \rightarrow W^{+} W^{-}$será denotado como $H \rightarrow W W, H \rightarrow \tau^{+} \tau^{-}$como $H \rightarrow \tau \tau$ e $H \rightarrow b \bar{b}$ como $H \rightarrow b b$.

Vários modos de decaimento sensíveis ao valor de $m_{H}$ foram estudados nos experimentos de 2011 no CMS a uma energia de centro de massa de $\sqrt{s}=7 \mathrm{TeV}$ porém, até o dia da divulgação de [12], apenas os cinco canais mais sensíveis haviam sido estudados a uma energia de $\sqrt{s}=8 \mathrm{TeV}$ no mesmo detector. $\mathrm{O}$ decaimento $H \rightarrow Z Z$ é seguido pelo decaimento de $Z Z$ em $4 l ; H \rightarrow \tau \tau$ seguido por pelo menos um decaimento leptônico do $\tau$; $H \rightarrow W W$ seguido pelo decaimento de $W W$ em $2 l 2 \nu$ e $H \rightarrow b b$ seguido pela fragmentação dos quarks $b$ em jatos.

Nos canais de estados finais $Z Z$ e $W W$, pelo menos um dos bósons $Z$ e $W$ está fora da camada de massa. Os resultados das buscas de 2012 à energia $\sqrt{s}=8 \mathrm{TeV}$ no CMS são comparados e combinados com os obtidos na busca a $\sqrt{s}=7 \mathrm{TeV}$ em [12]. Os resultados indicam que a partícula encontrada trata-se de um bóson com spin diferente de um. Um excesso de eventos acima do esperado foi observado para nova partícula com uma massa próxima de $125 \mathrm{GeV}$ consistente com a produção do bóson de Higgs do MP.

Estudos já haviam indicado que o canal $H \rightarrow \gamma \gamma$ seria um dos mais promissores na região de massas pequenas para o bóson de Higgs do MP [44]. A busca no CMS foi realizada num alcance que variou de 110 a $150 \mathrm{GeV}$. Neste canal, também foi observado um excesso de eventos próximo de $m_{H}=$ $125 \mathrm{GeV}$ tanto nos dados experimentais tomados a uma energia de $\sqrt{s}=7$ 
TeV quanto de $\sqrt{s}=8 \mathrm{TeV}$.

Nos canais com melhor resolução de massa: $\gamma \gamma$ e $Z Z$, observou-se um excesso mais significante em torno do valor $125 \mathrm{GeV}$. Os resultado foram apresentados em [12] e estão de acordo com as expectativas para o bóson de Higgs do MP. Porém, alguns resultados poderiam indicar ainda a possibilidade de acomodar física nova.

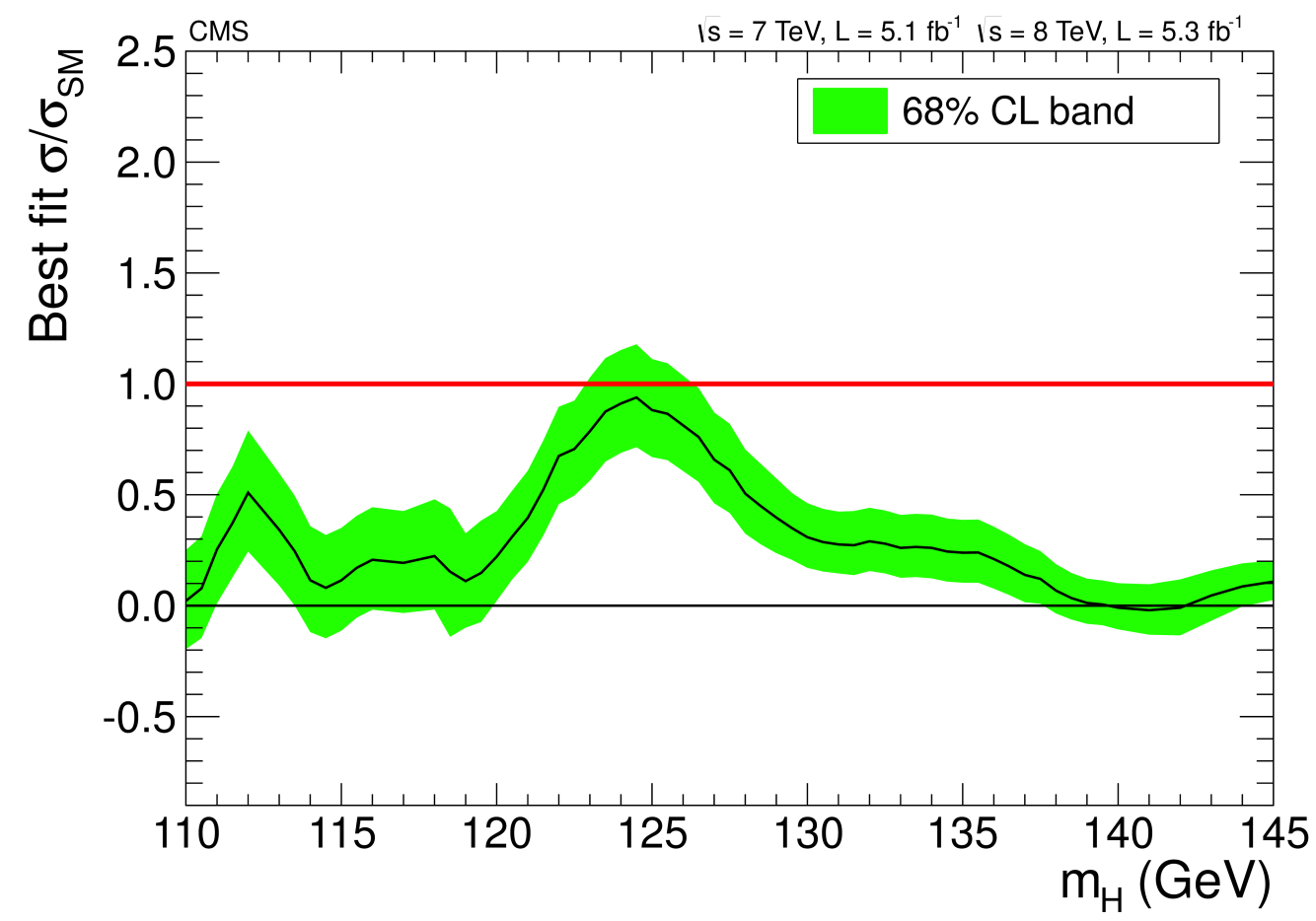

Figura 3: Intensidade do sinal definido como $\frac{\sigma}{\sigma_{M P}}$ em função da massa do bóson de Higgs para os dados combinados de medidas a energias de centrode-massa de $7 \mathrm{TeV}$ e $8 \mathrm{TeV}$. A faixa verde corresponde à região de erro compreendida entre $+\sigma$ e $-\sigma$ (desvio-padrão) das medidas [12].

A Figura 3 representa a relação $\frac{\sigma}{\sigma_{M P}}$ da seção de choque medida em todos os canais de decaimento combinados com a seção de choque estimada a partir de cálculos do MP em função da massa do bóson de Higgs. Este resultado não representa a medida mais atualizada para a relação. Os dados considerados correspondem aos obtidos no CMS a energias de centro-de-massa de $\sqrt{s}=7$ 
$\mathrm{TeV}$ e $\sqrt{s}=8 \mathrm{TeV}$ até 2012 [12]. Percebe-se que para valores de $m_{H}$ entre $123 \mathrm{GeV}$ e $127 \mathrm{GeV}$ a intensidade do sinal se aproxima de 1, o que indicou um intervalo provável para valores da massa.

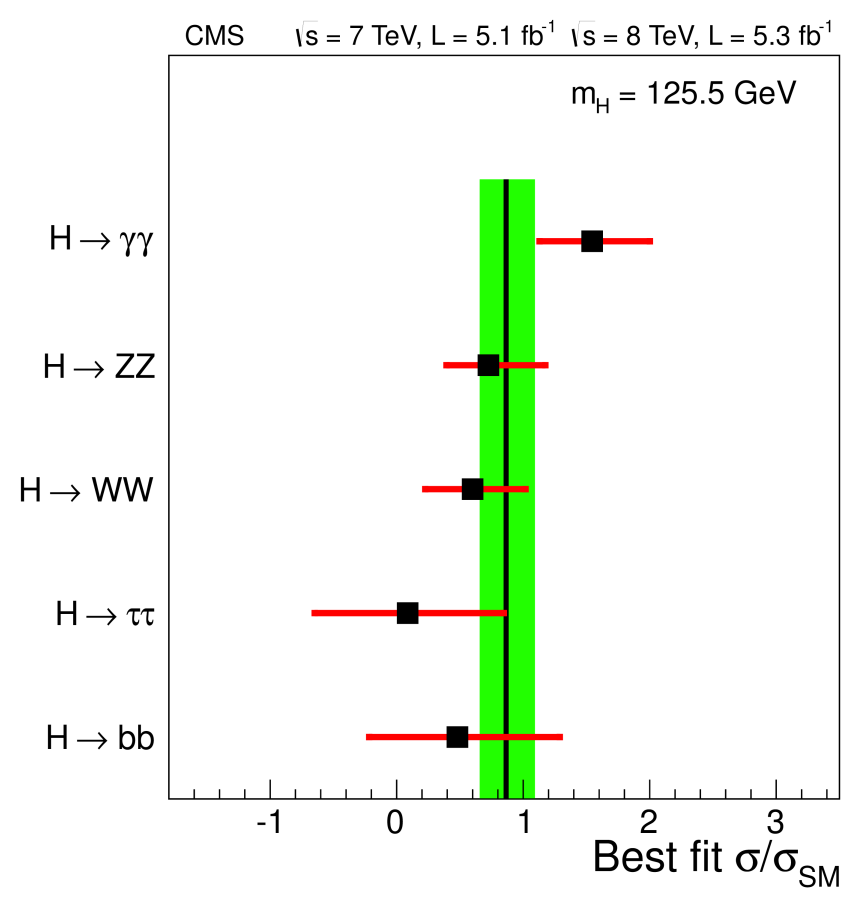

Figura 4: Intensidade do sinal $\frac{\sigma}{\sigma_{M P}}$ para cinco canais de decaimento medidos (pontos pretos) e para suas combinações (linha vertical preta). As barras horizontais vermelhas representam as regiões de $\pm \sigma$ em torno da média obtida para cada canal; a barra verde representa a região de $\pm 0,23$ em torno da média 0,87 dos canais combinados [12].

A Figura 4 representa a relação $\frac{\sigma}{\sigma_{M P}}$ para cada canal de decaimento $\gamma \gamma$, $Z Z, W W, \tau \tau$ e $b b$ e também para a combinação deles; usa-se o valor da massa do bóson de Higgs de $m_{H}=125,5 \mathrm{GeV}$. Um valor de 0,87 $\pm 0,23$ para intensidade combinada foi obtida [12]. Este valor não corresponde à medida atual obtida no CMS. Os dados considerados representam os obtidos no CMS a energias de centro-de-massa de $\sqrt{s}=7 \mathrm{TeV}$ e $\sqrt{s}=8 \mathrm{TeV}$ até 2012 . No decaimento $H \rightarrow \gamma \gamma$, o valor da intensidade do sinal obtido é $\mu=1,6 \pm 0,4$. Este resultado consideravelmente acima do previsto pelo MP pôde servir de 
inspiração para a busca de física nova.

\section{Resultados atualizados para o bóson de Higgs}

Nesta seção, serão apresentados alguns dos resultados mais recentes a respeito das pesquisas do bóson de Higgs nos detectores ATLAS e CMS. As análises de medidas combinadas nesses dois detectores foram feitas assumindo um valor de 125, $09 \mathrm{GeV}$ para a massa do bóson de Higgs em [45]. Os dados analisados foram obtidos em colisões próton-próton no LHC em 2011 e 2012.

\begin{tabular}{l|r|r|r}
\hline \hline Decay channel & ATLAS+CMS & ATLAS & \multicolumn{1}{c}{ CMS } \\
\hline$\mu^{\gamma \gamma}$ & $1.14_{-0.18}^{+0.19}$ & $1.14_{-0.25}^{+0.27}$ & $1.11_{-0.23}^{+0.25}$ \\
& $\left(\begin{array}{l}+0.18 \\
-0.17\end{array}\right)$ & $\left(_{-0.24}^{+0.26}\right)$ & $\left(\begin{array}{l}+0.23 \\
-0.21\end{array}\right)$ \\
$\mu^{Z Z}$ & $1.29_{-0.23}^{+0.26}$ & $1.52_{-0.34}^{+0.40}$ & $1.04_{-0.26}^{+0.32}$ \\
& $\left(\begin{array}{l}+0.23 \\
-0.20\end{array}\right)$ & $\left(_{-0.27}^{+0.32}\right)$ & $\left(_{-0.25}^{+0.30}\right.$ \\
$\mu^{W W}$ & $1.09_{-0.16}^{+0.18}$ & $1.22_{-0.21}^{+0.23}$ & $0.90_{-0.21}^{+0.23}$ \\
& $\left(\begin{array}{l}+0.16 \\
-0.15\end{array}\right)$ & $\left(_{-0.20}^{+0.21}\right)$ & $\left(\begin{array}{l}+0.23 \\
-0.20\end{array}\right)$ \\
$\mu^{\tau \tau}$ & $1.11_{-0.22}^{+0.24}$ & $1.41_{-0.36}^{+0.40}$ & $0.88_{-0.28}^{+0.30}$ \\
& $\left(\begin{array}{l}+0.24 \\
-0.22\end{array}\right)$ & $\left(\begin{array}{l}+0.37 \\
-0.33\end{array}\right)$ & $\left(\begin{array}{l}+0.31 \\
-0.29\end{array}\right)$ \\
$\mu^{b b}$ & $0.70_{-0.27}^{+0.29}$ & $0.62_{-0.37}^{+0.37}$ & $0.81_{-0.43}^{+0.45}$ \\
& $\left(\begin{array}{l}+0.29 \\
-0.28\end{array}\right)$ & $\left(\begin{array}{l}+0.39 \\
-0.37\end{array}\right)$ & $\left(\begin{array}{l}+0.45 \\
-0.43\end{array}\right)$ \\
$\mu^{\mu \mu}$ & $0.1_{-2.5}^{+2.5}$ & $-0.6_{-3.6}^{+3.6}$ & $0.9_{-3.5}^{+3.6}$ \\
& $\left(\begin{array}{l}+2.4 \\
-2.3\end{array}\right)$ & $\left(\begin{array}{l}+3.6 \\
-3.6\end{array}\right)$ & $\left(\begin{array}{l}+3.3 \\
-3.2\end{array}\right)$ \\
\hline \hline
\end{tabular}

Figura 5: Resultados atualizados para a intensidade do sinal de diversos canais (ATLAS+CMS) [45].

A Figura 5 apresenta as intensidades dos sinais medidos $(\mu)$ e suas incertezas para diferentes canais de decaimento do bóson de Higgs. Na segunda co- 
luna, mostramos os resultados combinados dos dois experimentos, enquanto que na terceira e quarta colunas hás os sinais obtidos em cada experiemnto separadamente. É importante ressaltar que o valor atual para $\mu$ do decaimento $H \rightarrow \gamma \gamma$ é de $1,14_{-0,18}^{+0,19}[45]$.

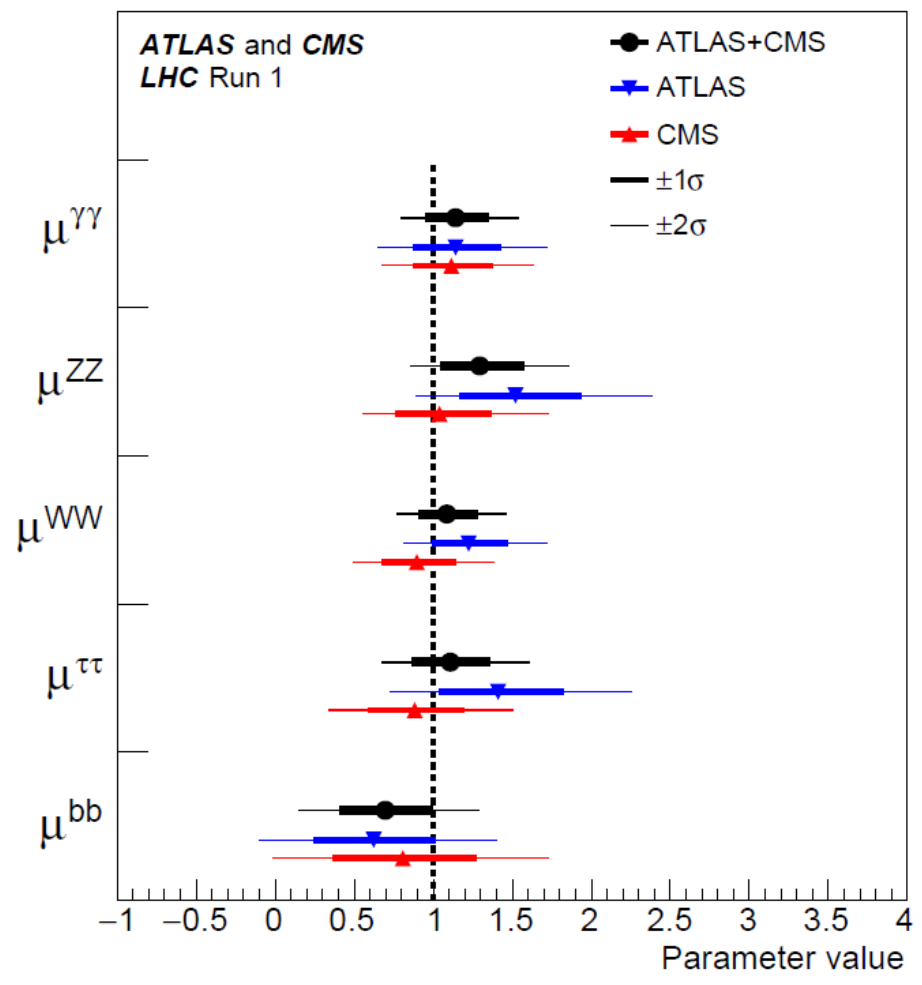

Figura 6: Ajuste dos resultados atualizados para a intensidade do sinal de diversos canais (ATLAS+CMS) e suas respectivas barras de erro $( \pm 1 \sigma)$ e $( \pm 2 \sigma)[45]$.

A Figura 6 apresenta o ajuste dos dados apresentados na Figura 5 em gráfico. As barras de erro indicam os intervalos de $1 \sigma$ (desvio-padrão), dadas pelas linhas grossas, e $2 \sigma$, dadas pelas linhas finas. Os decaimentos foram analisados em seis intensidades de sinais independentes, um para cada canal. É assumido que a seção de choque de produção do bóson de Higgs é a mesma da prevista pelo MP. 


\section{Novas pesquisas para o bóson de Higgs a partir do Run-2 do LHC}

A partir de 5 de Abril de 2015 as operações no LHC foram reiniciadas. Desta vez, dois feixes de prótons colidem a uma energia combinada de $\sqrt{s}=$

$13 \mathrm{TeV} ; 6,5 \mathrm{TeV}$ por feixe. Os dados obtidos nestas medidas servirão para refinar os resultados anteriores e buscar sinais de novas partículas. Até o presente momento, os resultados desta nova etapa de experimentos no LHC ainda não atingiram dados suficientes para competir com os resultados do run-1 (etapa compreendida entre 2011 e 2013).

\section{A proposta desta dissertação}

No Capítulo 1, apresentaremos como o mecanismo de Higgs atua no contexto do MP, justificando o aparecimento de termos de massa para campos massivos com a incorporação do bóson de Higgs. No Capítulo 2, utilizaremos as regras de Feynman para realizar o cálculo da largura de $H \rightarrow \gamma \gamma$ recorrendo à técnica da regularização dimensional.

Exibiremos como os loops de férmions, de bósons de gauge $W$ e de partículas escalares adicionais contribuem para a largura. Será mostrado também, o caso particular da contribuição do quark top e por que ela é dominante e suficiente para representar a contribuição das partículas de spin-1/2 na largura. Os resultados das contribuições vão depender das funções de loop, definidas para cada tipo de partícula.

Obteremos uma expressão para a largura com a adição de partículas novas e estimaremos o seu valor considerando apenas duas contribuições dominantes: do quark top e do bóson W. Compararemos este valor com o apresentado nos relatórios do CERN. Por fim, discutiremos como a adição de partículas novas podem alterar a largura do decaimento do Higgs em dois fótons. 


\section{Capítulo 1}

\section{Introdução ao modelo padrão eletrofraco}

O mecanismo que confere massa aos bósons de gauge $W^{ \pm}$e $Z^{0}$; e aos férmions fundamentais esteve entre os quesitos em aberto por décadas nas teorias propostas antes do advento do MP. Este mecanismo, que até pouco tempo não tinha sido testado experimentalmente, é conhecido como mecanismo da quebra espontânea da simetria eletrofraca (EWSB na sigla em inglês), ou mecanismo de Higgs.

O mecanismo de Higgs foi a proposta mais simples para o problema da EWSB e, desde que foi apresentada no começo dos anos 1960, foi tratada como parte do MPE antes mesmo da sua confirmação em 2012. Este mecanismo, quando aplicado ao contexto do MPE, introduz um dubleto de campos escalares complexos na lagrangeana original que se acopla com os campos do modelo. Como resultado desse campo adquirir um valor esperado de vácuo não nulo, bósons (com exceção do fóton) e férmions adquirem massas. $O$ preço desse mecanismo é a existência de uma nova partícula escalar: o bóson de Higgs. Ele seria a primeira e única partícula bosônica elementar que não transporta força de gauge.

Teorias além do MP tentam generalizar o que é feito pelo mecanismo de 
Higgs a fim de englobar simetrias mais inclusivas. O Modelo Supersimétrico Mínimo (MSM) é um exemplo onde dois pares de campos são incluídos na teoria primitiva dando origem a vários bósons de Higgs: dois escalares e um pseudoescalar neutros e um escalar carregado.

O anúncio da descoberta de uma partícula que se encaixa nas propriedades previstas para o bóson de Higgs [11, 12] simboliza um marco histórico para a física de partículas. A busca por este bóson no LHC recebeu especial atenção pela sua importância. Os resultados combinados para o valor de $m_{H}$ nos detectores ATLAS e CMS é hoje $m_{H}=(125,09 \pm 0,24) \mathrm{GeV}$ [46].

Todos esses resultados experimentais servem como prova aceita pela comunidade científica de que o MP é a teoria adequada para a descrição das interações eletrofracas e fortes [47]. Todas as peças que constituem esta teoria (as partículas previstas) foram confirmadas. No entanto, a possibilidade de existência de novas partículas ainda está em aberto e pode sugerir que o MP seja incompleto e necessite de extensões.

A invariância de gauge impede o surgimento de termos de massa para os bósons de gauge nas lagrangeanas de Yang-Mills. Tal característica é inadmissível na descrição de fenômenos que envolvem as interações fracas, onde observa-se que as partículas $W^{ \pm}$e $Z^{0}$ apresentam valores de massa bastante altos: $m_{W} \approx 80 \mathrm{GeV}$ e $m_{Z} \approx 91 \mathrm{GeV}$.

No entanto, em teorias de gauge como QED e QCD, cujos bósons de gauge (fótons e glúons, respectivamente) são desprovidos de massa, as lagrangeanas invariantes de gauge não apresentam problemas associados a essas simetrias. O mecanismo de Higgs no contexto do MP apresentou a solução mais plausível para a descrição das interações fracas, onde termos de massa surgem nas lagrangeanas para alguns dos bósons de gauge e não surgem para fótons e glúons.

A Tabela 1 fornece a atual conjuntura do que se conhece hoje da estrutura da matéria. As partículas mediadoras das interações são partículas vetoriais de spin 1, as partículas materiais são de spin 1/2 e o bóson de Higgs possui spin 0, sendo a única partícula elementar escalar conhecida. Quarks 
e léptons formam o conjunto das partículas materiais e diferem pelo fato de os primeiros participarem das interações fortes enquanto os segundos não.

\begin{tabular}{|c|c|c|}
\hline \multicolumn{3}{|c|}{ Tabela de partículas elementares } \\
\hline \multicolumn{3}{|c|}{ PARTÍCULAS MEDIADORAS } \\
\hline \multicolumn{2}{|c|}{ Interações Fortes } & Oito glúons \\
\hline \multicolumn{2}{|c|}{ Interações Eletromagnéticas } & Fóton $(\gamma)$ \\
\hline \multicolumn{2}{|c|}{ Interações Fracas } & Bósons $W^{ \pm}$e $Z^{0}$ \\
\hline \multicolumn{3}{|c|}{ PARTÍCULAS MATERIAIS } \\
\hline & Léptons & Quarks \\
\hline $1^{\mathrm{a}}$ Família & $\nu_{e}, e^{-}$ & $u, d$ \\
\hline $2^{\text {a }}$ Família & $\nu_{\mu}, \mu^{-}$ & $c, s$ \\
\hline $3^{a}$ Família & $\nu_{\tau}, \tau^{-}$ & $t, b$ \\
\hline \multicolumn{3}{|c|}{ BÓSON DE HIGGS } \\
\hline
\end{tabular}

Tabela 1.1: Atual estrutura da matéria conhecida.

\subsection{O setor de gauge no modelo padrão ele- trofraco}

A lagrangeana do setor de gauge do MPE possui a simetria do grupo $S U(2)_{L} \times U(1)_{Y}$. Este esqueleto compreende o ramo das interações fracas $\left(S U(2)_{L}\right)$ e da hipercarga $\left(U(1)_{Y}\right)$. A lagrangeana que reúne os termos cinéticos dos bósons de gauge é dada por:

$$
\mathcal{L}_{\text {gauge }}=-\frac{1}{4} B_{\mu \nu} B^{\mu \nu}-\frac{1}{4} W_{\mu \nu}^{a} W^{a \mu \nu},
$$

onde os tensores de campo são:

$$
\begin{array}{r}
B_{\mu \nu}=\partial_{\mu} B_{\nu}-\partial_{\nu} B_{\mu}, \quad \text { para a interação } U(1)_{Y} ; \\
W_{\mu \nu}^{a}=\partial_{\mu} W_{\nu}^{a}-\partial_{\nu} W_{\mu}^{a}+g \epsilon^{a b c} W_{\mu}^{b} W_{\nu}^{c},
\end{array}
$$


onde $g$ é uma constante de acoplamento associada ao grupo $S U(1)_{L}$ e os $\epsilon^{a b c}$ são as constantes de estrutura do grupo $S U(2)_{L}$. O tensor (1.2) obedece à simetria de grupo abeliano $U(1)_{Y}$ e possui a forma de gauge semelhante a do eletromagnetismo. Os índices $a, b$ e $c$ variam de 1 a 3 em (1.3). As constantes de estrutura dos grupos são definidas a partir do comutador entre geradores do grupo em questão:

$$
\left[T^{a}, T^{b}\right]=i \epsilon^{a b c} T^{c}
$$

onde os $T^{a}$ são as matrizes geradoras do grupo $S U(2)_{L}$.

A lagrangeana deve ser invariante sob as seguintes transformações nos campos de gauge:

$$
\begin{aligned}
B_{\mu} & \rightarrow B_{\mu}+\frac{1}{g^{\prime}} \partial_{\mu} \lambda_{Y}(x), \\
W_{\mu}^{a} & \rightarrow W_{\mu}^{a}+\frac{1}{g} \partial_{\mu} \lambda_{L}^{a}(x)+\epsilon^{a b c} W_{\mu}^{b} \lambda_{L}^{c}(x),
\end{aligned}
$$

onde $g^{\prime}$ é uma constante de acoplamento associada ao grupo $U(1)_{Y}$. A impossibilidade da existência de um termo de massa do tipo

$$
\frac{1}{2} m_{B}^{2} B_{\mu} B^{\mu}
$$

uma vez que esse termo quebra a invariância de gauge, a princípio, implica que todos estes bósons não possuem massa. A lagrangeana também deve ser invariante sob as seguintes transformações dos dubletos que interagem com os bósons da teoria:

$$
\begin{aligned}
& \psi \rightarrow e^{i \lambda_{Y}(x) Y} \psi, \\
& \psi \rightarrow e^{i \lambda_{L}^{a}(x) T^{a}} \psi,
\end{aligned}
$$

onde $Y$ é o operador hipercarga fraca.

Por fim, as interações entre os bósons de gauge e as partículas bosônicas ou fermiônicas da teoria são obtidas com o uso da seguinte derivada covariante

$$
\mathcal{D}_{\mu}=\partial_{\mu}-i g^{\prime} B_{\mu} Y-i g W_{\mu}^{a} T^{a}
$$




\subsection{O mecanismo de Higgs no modelo padrão eletrofraco}

No MPE, um dubleto escalar complexo é incorporado à teoria com a finalidade de se obter graus de liberdades adicionais para os campos de gauge por meio da aplicação do mecanismo de Higgs [1, 2, 3]. Sabe-se que os bósons de gauge sem massa possuem duas polarizações possíveis e que devemos ter uma polarização adicional para bósons massivos.

Considere o dubleto $\Phi$ de campos escalares:

$$
\Phi(x) \equiv\left(\begin{array}{c}
\phi^{+}(x) \\
\phi^{0}(x)
\end{array}\right)=\frac{1}{\sqrt{2}}\left(\begin{array}{c}
\phi_{1}(x)+i \phi_{2}(x) \\
\phi_{3}(x)+i \phi_{4}(x)
\end{array}\right) .
$$

Atribui-se o valor $Y_{\Phi}=\frac{1}{2}$ para a hipercarga de $\Phi$. Vamos adicionar à teoria a langrangeana que envolve estes campos adicionais:

$$
\mathcal{L}_{\Phi}=\left(\mathcal{D}^{\mu} \Phi\right)^{\dagger} \mathcal{D}_{\mu} \Phi-\lambda\left(|\Phi|^{2}-\frac{v^{2}}{2}\right)^{2}+\mathcal{L}_{\text {Yukawa }}
$$

onde a derivada covariante $\mathcal{D}_{\mu}=\partial_{\mu}-i g^{\prime} B_{\mu} Y_{\Phi}-\frac{i}{2} g \sigma^{a} W_{\mu}^{a}$ é responsável por acoplar o dubleto escalar com os bósons de gauge; e $\mathcal{L}_{\text {Yukawa }}$ acopla o campo escalar com uma dupla de férmions quirais. O termo do potencial nesta lagrangeana é o que provoca a quebra espontânea da simetria.

Primeiramente, vamos examinar o potencial de (1.12) dado por

$$
V(\Phi)=\lambda\left(|\Phi|^{2}-\frac{v^{2}}{2}\right)^{2}, v^{2}>0 .
$$

Se $\lambda$ for negativo, então o potencial é ilimitado para valores negativos de energia, portanto não é de nosso interesse essa situação. Se $\lambda$ for positivo, então um conjunto infinito de estados degenerados de mínima energia e que não são invariantes sob rotações do tipo $S U(2)_{L} \times U(1)_{Y}$ serão possíveis. Neste caso, os estados de mínima energia são os que obedecem a condição $|\Phi|=\frac{v}{\sqrt{2}}$. 
Tratando $|\Phi|^{2}$ em termos dos quatro graus de liberdade reais dados em (1.11) nota-se que este valor pode ser visto como o módulo quadrado de um vetor de quatro componentes:

$$
|\Phi|^{2}=\frac{1}{2}\left(\phi_{1}^{2}+\phi_{2}^{2}+\phi_{3}^{2}+\phi_{4}^{2}\right) .
$$

Portanto, os estados de vácuo formam um conjunto que pode ser representado por uma esfera de quatro dimensões.

O potencial $V$ pode também ser exibido em termos dos quatro escalares reais:

$$
V=\frac{\lambda}{4}\left(\phi^{2}{ }_{1}+\phi_{2}^{2}+\phi^{2}{ }_{3}+\phi^{2}{ }_{4}-v^{2}\right)^{2}, v^{2}>0 .
$$

Podemos escolher um estado arbitrário para representar o valor esperado de vácuo, em particular quando:

$$
\left\langle\phi_{3}\right\rangle \equiv v,\left\langle\phi_{1}\right\rangle=\left\langle\phi_{2}\right\rangle=\left\langle\phi_{4}\right\rangle=0 .
$$

Podemos agora redefinir $\Phi$ como:

$$
\Phi(x)=\frac{1}{\sqrt{2}}\left(\begin{array}{c}
\phi_{1}(x)+i \phi_{2}(x) \\
v+H(x)+i \phi_{4}(x)
\end{array}\right),
$$

onde $H(x)$ é um campo escalar, chamado de campo de Higgs, cujo valor esperado de vácuo é $\langle H(x)\rangle=0$.

Substitui-se em (1.13) o campo $\Phi$ na forma acima, obtendo:

$$
V=\frac{\lambda}{4}\left(\phi_{1}^{2}+\phi_{2}^{2}+(v+H)^{2}+\phi^{2}{ }_{4}-v^{2}\right)^{2}, v^{2}>0 .
$$

Expandindo a expressão acima termo a termo, obtemos:

$V=\frac{\lambda}{4}\left(\phi_{1}^{4}+\phi_{2}^{4}+4 v^{2} H^{2}+H^{4}+\phi_{4}^{4}+4 v H^{3}+\right.$ interações entre os campos $)$.

Observa-se que nenhum termo de massa aparece, com exceção de $\lambda v^{2} H^{2}$ indicando o valor $m_{H}=\sqrt{2 \lambda v^{2}}$ para a massa do bóson de Higgs. O valor conhecido experimentalmente para $v$ é de aproximadamente $246 \mathrm{GeV}$.

O campo $\Phi$ pode também ser manifestado como uma rotação do tipo $S U(2)_{L}$ de um estado dado como uma variação em torno de um dos estados 
de vácuo degenerados. Escolhendo o estado de mínima energia que obedece as condições dadas em (1.16) e tratando o campo $H(x)$ como uma variação em torno deste estado, temos que $\Phi(x)$ pode ser representado como:

$$
\Phi(x)=\exp \left(\frac{i}{v} \vec{\xi}(x) \cdot \vec{\sigma}\right) \frac{1}{\sqrt{2}}\left[\begin{array}{c}
0 \\
v+H(x)
\end{array}\right],
$$

onde os geradores do grupo $S U(2)_{L}$ são $\vec{T}=\frac{\vec{\sigma}}{2}$, sendo $\vec{\sigma}$ as matrizes de Pauli.

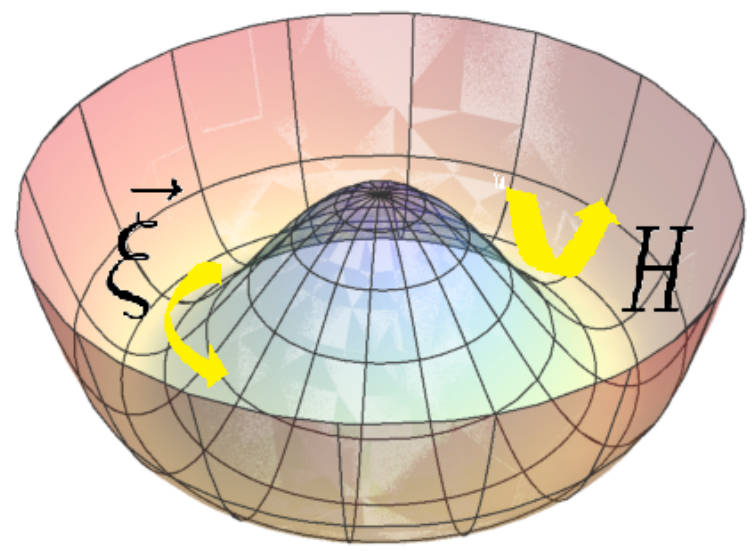

Figura 1.1: O potencial do campo $\Phi$ na situação de quebra espontânea de simetria. As setas indicam como os campos $\vec{\xi}(x)$ de Goldstone e $H(x)$ de Higgs parametrizam as excitações em torno de um estado de mínima energia [48].

A Figura 1.1 representa o potencial para $\Phi$ na situação onde é possível ocorrer uma infinidade de estados de mínima energia. Os parâmetros $\vec{\xi}(x)$ e $H(x)$ excitam o estado em torno de um mínimo escolhido, como se vê em (1.20). As setas indicam as direções ao longo das quais o estado se excita sob a influência de cada parâmetro.

Como visto em (1.9), o campo $\Phi$ pode sofrer uma transformação do tipo

$$
\Phi \rightarrow \exp \left(\frac{i}{2} \vec{\lambda}_{L}(x) \cdot \vec{\sigma}\right) \Phi,
$$

e manter invariância de gauge. Convenientemente, o gauge $\overrightarrow{\lambda_{L}}=\frac{-2 \vec{\xi}}{v}$ conhecido como gauge unitário é escolhido. Nele, os três campos sem massa 
observados em (1.19) são removidos e o primeiro termo à direita da igualdade em (1.12) fica:

$$
\begin{aligned}
\left(\mathcal{D}^{\mu} \Phi\right)^{\dagger} \mathcal{D}_{\mu} \Phi & =\frac{1}{2}\left(\partial_{\mu} H \partial^{\mu} H+\frac{g^{2}}{4}(v+H)^{2}\left(W^{1 \mu}-i W^{2 \mu}\right)\left(W_{\mu}^{1}+i W_{\mu}^{2}\right)+\right. \\
& \left.+\left(\frac{g}{2} W_{\mu}^{3}-g^{\prime} B_{\mu} Y_{\Phi}\right)\left(\frac{g}{2} W^{3 \mu}-g^{\prime} B^{\mu} Y_{\Phi}\right)(v+H)^{2}\right) .
\end{aligned}
$$

Após definir novos campos $W_{\mu}^{ \pm}, Z_{\mu}$ e $A_{\mu}$ em termos de $B_{\mu}$ e $W_{\mu}^{a}$ de forma apropriada, obtemos termos de massa $\frac{1}{2} m_{W}^{2} W_{\mu}^{\dagger} W^{\mu}+\frac{1}{2} m_{Z}^{2} Z_{\mu} Z^{\mu}$ e termos de interações. Os acoplamentos do tipo $H W_{\mu}^{\dagger} W^{\mu}$ e $H Z_{\mu} Z^{\mu}$ são proporcionais às massas ao quadrado $m_{W}^{2}$ e $m_{Z}^{2}$, respectivamente.

Os campos $Z_{\mu}$ e $A_{\mu}$ podem ser definidos a partir de $W_{\mu}^{3}$ e $B_{\mu}$ por meio da seguinte rotação:

$$
\left(\begin{array}{c}
W_{\mu}^{3} \\
B_{\mu}
\end{array}\right)=\left(\begin{array}{cc}
\cos \theta_{W} & \sin \theta_{W} \\
-\sin \theta_{W} & \cos \theta_{W}
\end{array}\right)\left(\begin{array}{c}
Z_{\mu} \\
A_{\mu}
\end{array}\right),
$$

onde $\theta_{W}$ é conhecido como ângulo de mistura fraca. As massas de $W$ e $Z$ e a carga elétrica elementar $e$ podem ser dadas em termos de $v, g, g^{\prime}$ e $\theta_{W}$ : $m_{W}=m_{Z} \cos \theta_{W}=\frac{1}{2} g v$ e $e=g \sin \theta_{W}=g^{\prime} \cos \theta_{W}$. Resultados experimentais indicam que o valor do ângulo de mistura fraca obedece à igualdade $\sin ^{2} \theta_{W}=$ $0,223[49]$.

Os bósons de gauge, que anteriormente à aplicação do mecanismo de Higgs possuíam duas polarizações, adquirem mais uma polarização longitudinal ao preço de eliminar três bósons de Goldstone. É comum referir-se ao caso como: os bósons de gauge "engolem" os bósons de Goldstone. O número de graus de liberdade (gdl) permanece o mesmo: um gdl de cada um dos três bósons de Goldstone são transferidos para cada bóson de gauge.

Termos de auto-interação cúbica e quártica do campo escalar $H$ são obtidos do potencial de (1.12), assim como o seu termo de massa. O termo cinético $\frac{1}{2} \partial_{\mu} H \partial^{\mu} H$ aparece em (1.22). No gauge unitário, temos que:

$$
-\lambda\left(|\Phi|^{2}-\frac{v^{2}}{2}\right)^{2}=-\frac{\lambda}{4} H^{4}-\lambda v H^{3}-\lambda v^{2} H^{2} .
$$


Portanto, definindo $m_{H}^{2}=2 \lambda v^{2}$ obtemos a lagrangeana para o campo de Higgs livre e com suas auto interações:

$$
\mathcal{L}_{H}=\frac{1}{2} \partial_{\mu} H \partial^{\mu} H-\frac{1}{2} m_{H}^{2} H^{2}-\frac{m_{H}^{2}}{2 v} H^{3}-\frac{m_{H}^{2}}{8 v^{2}} H^{4} .
$$

Com o valor de $m_{H}=125,09 \mathrm{GeV}$ obtido nos experimentos de 2012 no LHC $[11,12,46]$ encontramos o valor da constante $\lambda$ :

$$
\lambda=0,13 .
$$

\subsection{Considerações sobre o setor fermiônico no modelo padrão eletrofraco}

Uma família de férmions fundamentais manifestam-se como campos quirais sob as transformações de gauge do tipo $S U(2)_{L} \times U(1)_{Y}$ do MP. Os operadores de projeção $P_{R}$ e $P_{L}$ são definidos como operadores que nos fornecem as projeções quirais, chamadas de estados de mão-direita e de mão-esquerda a partir de um spinor de Dirac:

$$
P_{R} \psi \equiv \psi_{R}, \quad P_{L} \psi \equiv \psi_{L}
$$

onde valem as relações

$$
\begin{array}{cc}
\bar{\psi} P_{R}=\bar{\psi}_{L}, \quad \bar{\psi} P_{L}=\bar{\psi}_{R}, \\
P_{R}^{2}=P_{R}, \quad P_{L}^{2}=P_{L} \mathrm{e} \\
P_{R}+P_{L}=1 . &
\end{array}
$$

Os operadores que obedecem a estas propriedades são definidos como:

$$
P_{R}=\frac{1}{2}\left(1+\gamma^{5}\right), \quad P_{L}=\frac{1}{2}\left(1-\gamma^{5}\right) .
$$

A partir destas propriedades dos operadores de projeção podemos denotar a lagrangenana de um campo de Dirac, $\mathcal{L}=\bar{\psi} i \partial_{\mu} \gamma^{\mu} \psi-m \bar{\psi} \psi$, em termos dos campos quirais. O termo cinético fica:

$$
\bar{\psi} i \partial_{\mu} \gamma^{\mu} \psi=\bar{\psi}_{L} i \partial_{\mu} \gamma^{\mu} \psi_{L}+\bar{\psi}_{R} i \partial_{\mu} \gamma^{\mu} \psi_{R}
$$


e o termo de massa:

$$
-m \bar{\psi} \psi=-m \bar{\psi}_{R} \psi_{L}-m \bar{\psi}_{L} \psi_{R}
$$

Percebe-se que o termo (1.32) é invariante de gauge, enquanto que o termo (1.33) não o é. Portanto, um termo de massa para os férmions não pode ser adicionada à teoria, implicando a ausência de massa para os férmions no MP antes da quebra da simetria.

Em cada família, as componentes de mão-esquerda dos campos fermiônicos aparecem em dubletos de $S U(2)_{L} \times U(1)_{Y}$ enquanto as componentes de mãodireita aparecem em singletos. Não consideraremos aqui: neutrinos de mãodireita. O setor fermiônico é representado por três famílias de quarks e léptons quirais. Em cada dubleto há um lépton $\left(l^{-}\right)$e seu neutrino correspondente $\left(\nu_{l}\right)$ ou um quark up $\left(q_{u}\right)$ e um quark down $\left(q_{d}\right)$ representados por:

$$
\left[\begin{array}{cc}
\nu_{l} & q_{u} \\
l^{-} & q_{d}
\end{array}\right] \equiv\left(\begin{array}{c}
\nu_{l L} \\
l_{L}^{-}
\end{array}\right),\left(\begin{array}{c}
q_{u L} \\
q_{d L}
\end{array}\right), l_{R}^{-}, q_{u R}, q_{d R},
$$

cada uma das famílias, ou seja, para as três famílias do MP temos:

$$
\left[\begin{array}{ll}
\nu_{e} & u \\
e^{-} & d
\end{array}\right],\left[\begin{array}{ll}
\nu_{\mu} & c \\
\mu^{-} & s
\end{array}\right],\left[\begin{array}{ll}
\nu_{\tau} & t \\
\tau^{-} & b
\end{array}\right] .
$$

Para resolver a questão da impossibilidade de existência de massa para os férmions, adiciona-se uma lagrangeana invariante de gauge e que acopla os férmions ao dubleto escalar complexo $\Phi$ :

$$
\mathcal{L}_{Y u k}=-y_{d} \bar{Q}_{L} \Phi d_{R}-y_{u} \bar{Q}_{L} \tilde{\Phi} u_{R}-y_{l} \bar{L}_{L} \Phi l_{R}+\text { h.c. },
$$

onde $\tilde{\Phi} \equiv i \sigma_{2} \Phi^{*} ; y_{d}, y_{u}$ e $y_{l}$ são matrizes de parâmetros livres. Os dubletos de quarks e léptons de mão-esquerda são representados por $\bar{Q}_{L}=\left(\begin{array}{ll}\bar{u}_{L} & \bar{d}_{L}\end{array}\right)$ e $\bar{L}_{L}=\left(\begin{array}{ll}\bar{\nu}_{L} & \bar{l}_{L}\end{array}\right)$, respectivamente; e os correspondentes singletos de mãodireita são retratados como $u_{R}, d_{R}$ e $l_{R}$.

Após a diagonalização de (1.36), podemos escrever de forma explícita no 
gauge unitário para o dubleto $\Phi$ a seguinte expressão:

$$
\begin{aligned}
\mathcal{L}_{Y u k} & =-\sum_{d=d, s, b} \frac{\tilde{y}_{d}}{\sqrt{2}}(v+H)\left(\bar{d}_{L} d_{R}+\bar{d}_{R} d_{L}\right)-\sum_{u=u, c, t} \frac{\tilde{y}_{u}}{\sqrt{2}}(v+H)\left(\bar{u}_{L} u_{R}+\bar{u}_{R} u_{L}\right)+ \\
& -\sum_{l=e, \mu, \tau} \frac{\tilde{y}_{l}}{\sqrt{2}}(v+H)\left(\bar{l}_{L} l_{R}+\bar{l}_{R} l_{L}\right) .
\end{aligned}
$$

Definindo

$$
m_{f} \equiv \tilde{y}_{f} \frac{v}{\sqrt{2}}, \text { para } f=d, s, b, u, c, t, e, \mu, \tau
$$

e usando a relação

$$
\bar{\psi} \psi=\bar{\psi}_{L} \psi_{R}+\bar{\psi}_{R} \psi_{L}
$$

onde $\psi$ representa um campo de Dirac genérico; temos que a lagrangeana (1.36) adquire a seguinte forma:

$$
\mathcal{L}_{Y u k}=-\left(1+\frac{H}{v}\right)\left(\sum_{d=d, s, b} m_{d} \bar{d} d+\sum_{u=u, c, t} m_{u} \bar{u} u+\sum_{l=e, \mu, \tau} m_{l} \bar{l} l\right) .
$$

O mecanismo de Higgs e a EWSB no contexto do MP contribui assim para o surgimento de massa para as partículas fermiônicas. Os acoplamentos $\tilde{y}_{f}$ são, portanto dados em termos das massas dos férmions, que são determinadas experimentalmente. Desta forma, esses parâmetros são de fato parâmetros livres da teoria. 


\section{Capítulo 2}

\section{A largura de decaimento $H \rightarrow \gamma \gamma$ e as diversas contribuições possíveis}

A largura de decaimento do bóson de Higgs em dois fótons pode ser calculada por meio da utilização das regras de Feynman. Por não se acoplar diretamente ao fóton, o bóson não decai em dois fótons a nível de árvore. Para descobrir uma expressão para a largura, calcula-se as contribuições de 1-loop na amplitude devido à presença de férmions, bósons de gauge $\mathrm{W}$ e partículas escalares.

No MP há apenas a contribuição do bóson $\mathrm{W}$ e dos férmions do modelo para essa largura, mas em modelos além do MP é possível haver contribuições de novas partículas, em particular, de um novo escalar. Por essa razão, incluímos aqui também esta contribuição. Além desta partícula escalar, outra partícula fermiônica genérica poderia ser adicionada. Os resultados apresentados a seguir são resumos dos extensos cálculos realizados.

Classicamente, a medida da largura de decaimento de uma partícula é dada por:

$$
\Gamma=\frac{\text { número de decaimentos }}{\text { tempo de medida } \cdot \text { densidade de partículas iniciais }} .
$$


Em mecânica quântica, no entanto, a largura de decaimento é calculada em termos da densidade de probabilidade de tal processo ocorrer. Portanto, a taxa de decaimento diferencial é:

$$
d \Gamma=\frac{1}{T} d P
$$

onde $T$ é o tempo do processo e $d P$ é a densidade de probabilidade.

Dado dois estados $|i\rangle$ e $|f\rangle$ representando estados de um sistema antes e depois de um decaimento, respectivamente, temos que a densidade de probabilidade de um processo levar um estado a outro em teoria quântica de campos é:

$$
d P=\frac{|\langle f|S| i\rangle|^{2}}{\langle f \mid f\rangle\langle i \mid i\rangle} \prod_{j} \frac{V}{(2 \pi)^{3}} d^{3} p_{j},
$$

onde $S$ é a conhecida matriz- $S$ unitária que conecta os estados assintóticos final e inicial de um sistema físico que sofre espalhamento, $V$ é o volume total da região do processo (formalmente infinito, mas cujo valor não influencia no fim do cálculo) e $p_{j}$ é o momento da partícula $j$ no estado final. Os termos de normalização $\langle i \mid i\rangle$ e $\langle f \mid f\rangle$ são:

$$
\langle i \mid i\rangle=2 E_{i} V \text { e }\langle f \mid f\rangle=\prod_{j}\left(2 E_{j} V\right)
$$

onde $E_{i}$ e $E_{j}$ representam as energias da partícula inicial e da partícula $j$ final, respectivamente.

Podemos expressar a matrix $S$ em termo da amplitude de espalhamento $\mathcal{M}:\langle f|(S-1)| i\rangle=(2 \pi)^{4} \delta^{(4)}\left(p_{i}-\sum p_{j}\right) \cdot i \mathcal{M}$.

$$
d \Gamma=\frac{|\mathcal{M}|^{2}}{2 E_{i}} \delta^{(4)}\left(p_{i}-\sum_{j} p_{j}\right)(2 \pi)^{4} \prod_{j} \frac{d^{3} p_{j}}{(2 \pi)^{3}\left(2 E_{j}\right)} .
$$

Para o caso do decaimento do bóson de Higgs em dois fótons, temos $j=1$ e 2 , identificando cada um dos fótons finais. Como trata-se de duas partículas idênticas devemos dividir o resultado final da expressão acima por 2, que serve como peso estatístico que elimina as contagens repetidas de partículas. Integrando a expressão acima em todo o espaço dos momentos dos fótons 
finais no referencial do centro de massa (c.m.), obtemos uma expressão para $d \Gamma$ em termo do ângulo sólido diferencial $d \Omega_{c . m}$. para o decaimento $H \rightarrow \gamma \gamma$ :

$$
\frac{d \Gamma}{d \Omega_{c . m .}}=\frac{|\mathcal{M}|^{2}}{128 \pi^{2}} \frac{1}{m_{H}} .
$$

Integrando a expressão (2.6) em $d \Omega_{\text {c.m. }}$. encontramos a largura total do decaimento $H \rightarrow \gamma \gamma$ :

$$
\Gamma=\frac{|\mathcal{M}|^{2}}{32 \pi m_{H}} .
$$

As regras de Feynman úteis para a determinação das amplitudes do decaimento são as seguintes:
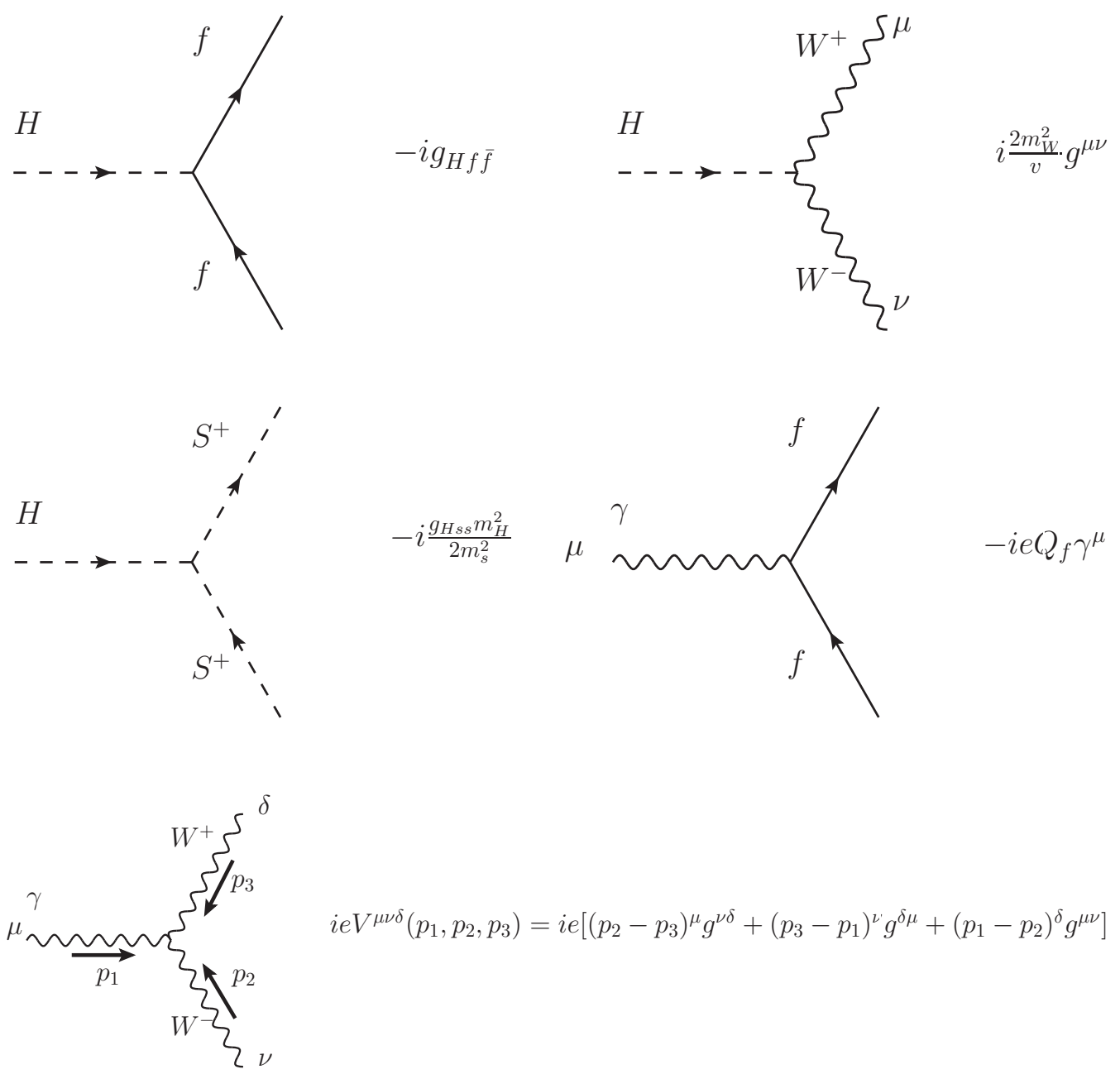

$i e V^{\mu \nu \delta}\left(p_{1}, p_{2}, p_{3}\right)=i e\left[\left(p_{2}-p_{3}\right)^{\mu} g^{\nu \delta}+\left(p_{3}-p_{1}\right)^{\nu} g^{\delta \mu}+\left(p_{1}-p_{2}\right)^{\delta} g^{\mu \nu}\right]$ 

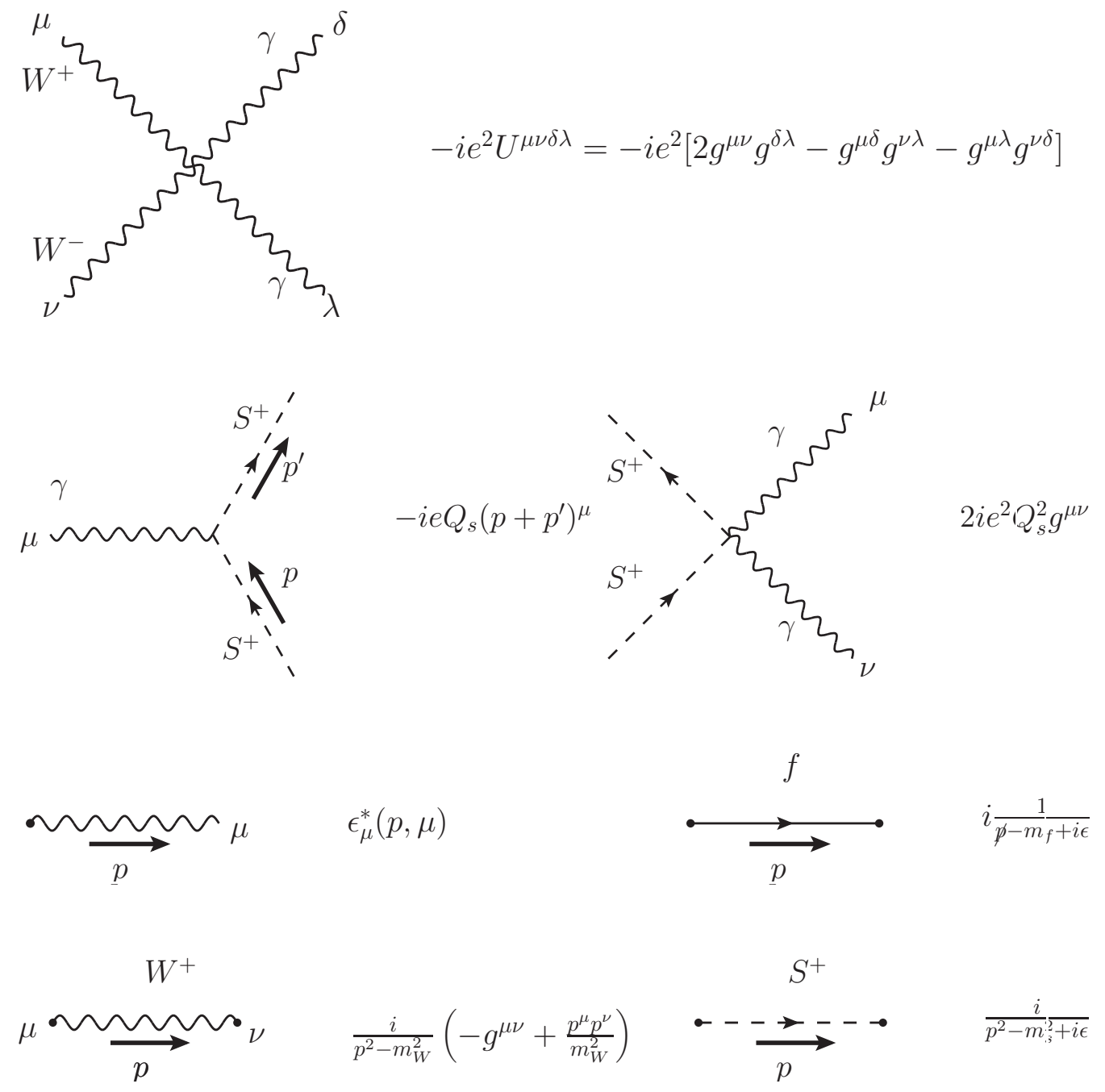

As constantes de acoplamento $g_{H f \bar{f}}$ e $v$ podem ser representadas em termo da constante $g$ da teoria eletrofaca da seguinte maneira:

$$
\begin{aligned}
g_{H f \bar{f}} & \equiv \frac{g m_{f}}{2 m_{W}} \\
v & \equiv \frac{2 m_{W}}{g}
\end{aligned}
$$

Iniciaremos apresentando as contribuições presentes no MP: contribuição dos férmions quirais e bósons W. Por último, será apresentada a contribuição referente às partículas escalares. 


\subsection{A contribuição dos loops de férmions no decaimento $H \rightarrow \gamma \gamma$}

A seguir, temos os diagramas de Feynman da contribuição fermiônica em ordem mais baixa da teoria de perturbações para o decaimento $H \rightarrow \gamma \gamma$ :
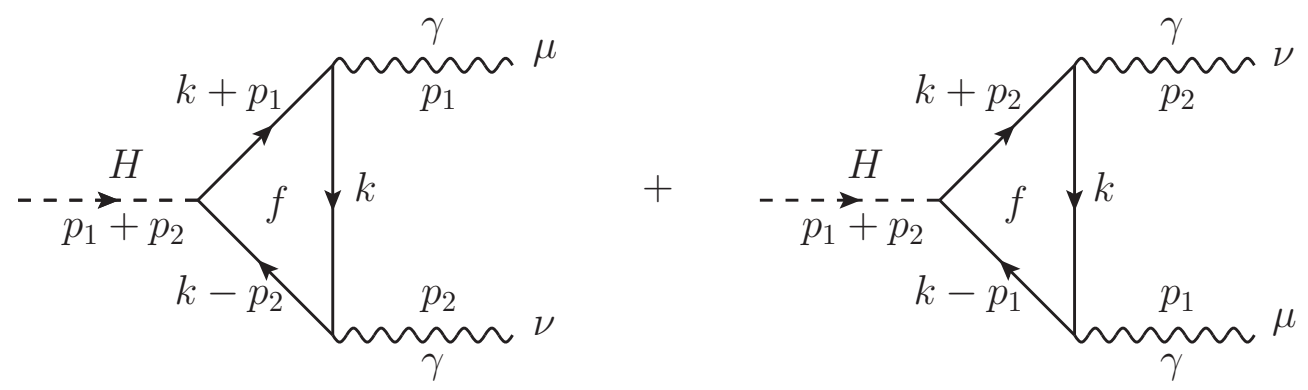

Seguindo as regras de Feynman apresentadas acima, temos que a amplitude para a contribuição fermiônica é:

$$
\begin{aligned}
\mathcal{M}_{f} & =i g_{H f \bar{f}} \epsilon_{\mu}^{*}\left(p_{1}\right) \epsilon_{\nu}^{*}\left(p_{2}\right) \times \\
& \times \int \frac{d^{4} k}{(2 \pi)^{4}}\left(\frac{\operatorname{tr}\left[i\left(k+\not{ }_{1}+m_{f}\right)\left(i Q_{f} e \gamma^{\mu}\right) i\left(k+m_{f}\right)\left(i Q_{f} e \gamma^{\nu}\right) i\left(k-\not p_{2}+m_{f}\right)\right]}{\left[\left(k+p_{1}\right)^{2}-m_{f}^{2}\right]\left[k^{2}-m_{f}^{2}\right]\left[\left(k-p_{2}\right)^{2}-m_{f}^{2}\right]}+\right. \\
& \left.+\frac{\operatorname{tr}\left[i\left(k+\not m_{f}\right)\left(i Q_{f} e \gamma^{\nu}\right) i\left(k+m_{f}\right)\left(i Q_{f} e \gamma^{\mu}\right) i\left(k-\not p_{1}+m_{f}\right)\right]}{\left[\left(k+p_{2}\right)^{2}-m_{f}^{2}\right]\left[k^{2}-m_{f}^{2}\right]\left[\left(k-p_{1}\right)^{2}-m_{f}^{2}\right]}\right)
\end{aligned}
$$

Como o vértice envolvendo um fóton e dois férmions contém uma matriz $\gamma^{\mu}$, necessita-se calcular na expressão acima o traço do produto de diversas matrizes $\gamma$. Para tanto, utiliza-se as propriedades (A.1-A.4) da seção A.1 do Apêndice A. Por exemplo, um dos termos que surgem no numerador interno à integral é $m_{f} k_{\alpha} k_{\beta} \operatorname{tr}\left[\gamma^{\alpha} \gamma^{\beta} \gamma^{\nu} \gamma^{\mu}\right]$, cujo valor é $4 m_{f} k^{2} g^{\mu \nu}$.

Outra técnica bastante utilizada em cálculos de integrais de loop é a parametrização de Feynman. Nela, procuramos um forma de simplificar frações 
do integrando do tipo que aparece em (2.10), por exemplo:

$$
\frac{1}{\left[\left(k+p_{1}\right)^{2}-m_{f}^{2}\right]\left[k^{2}-m_{f}^{2}\right]\left[\left(k-p_{2}\right)^{2}-m_{f}^{2}\right]} .
$$

Com esta técnica, consegue-se expressar o denominador do integrando em um formato ideal para aplicar a regularização dimensional. Resultados gerais da parametrização de Feynman úteis para a resolução deste tipo de problema estão resumidos na seção A.2 do Apêndice A.

Ao expressar (2.11) em termos dos parâmetros de Feynman, obtemos o resultado (A.12) da seção A.2 do Apêndice A. Substituindo esta solução em (2.10) e invertendo a ordem de integração, obtemos uma soma de termos proporcionais a:

$$
\begin{aligned}
& \int_{0}^{1} d u \int_{0}^{1-u} d w \int \frac{d^{4} x}{(2 \pi)^{4}} \frac{1}{\left[x^{2}-\Lambda\right]^{3}} \\
& \int_{0}^{1} d u \int_{0}^{1-u} d w \int \frac{d^{4} x}{(2 \pi)^{4}} \frac{x^{2}}{\left[x^{2}-\Lambda\right]^{3}} \mathrm{e} \\
& \int_{0}^{1} d u \int_{0}^{1-u} d w \int \frac{d^{4} x}{(2 \pi)^{4}} \frac{x^{\mu} x^{\nu}}{\left[x^{2}-\Lambda\right]^{3}}
\end{aligned}
$$

As integrais na variável $x$ podem ser calculadas por meio da regularização dimensional, outra técnica bastante usada em cálculos envolvendo loops, cujas soluções são apresentadas na seção A.3 do Apêndice A. As técnicas apresentadas nesta seção serão utilizada nas contribuições seguintes de maneira análoga.

A importância da aplicabilidade desta regularização nesta e nas outras contribuições vem do fato que a amplitude (2.10) (e as seguintes) apresentam divergências logarítmicas. No entando, a aplicação da regularização dimensional permite que organizemos os termos divergentes de forma que é possível mostrar que todos se cancelem ao final.

O resultado final da contribuição fermiônica após a resolução das integrais de loop e a soma sobre as polarizações dos fótons finais é, portanto:

$$
\mathcal{M}_{f}=-i \frac{\alpha m_{H}^{2}}{4 \sqrt{2} \pi} \frac{2 g_{H f \bar{f}}}{m_{f}} N_{c f} Q_{f}^{2}\left(-2 \tau_{f}\left[1+\left(1-\tau_{f}\right) f\left(\tau_{f}\right)\right]\right),
$$


onde $\alpha$ é a constante de estrutura fina, $Q_{f}$ é a carga do férmion $f, N_{c f}$ é o número de cores do férmion ( $N_{c f}=1$ para léptons e $N_{c f}=3$ para quarks), $\tau_{f}=\frac{4 m_{f}^{2}}{m_{H}^{2}}$ e a função $f(\tau)$ definida da seguinte maneira:

$$
f(\tau)= \begin{cases}\left(\sin ^{-1}\left(\sqrt{\frac{1}{\tau}}\right)\right)^{2}, & \text { para } \tau \geq 1, \\ -\frac{1}{4}\left(\ln \left(\frac{\eta_{+}}{\eta_{-}}\right)-i \pi\right)^{2}, & \text { para } \tau<1\end{cases}
$$

onde

$$
\eta_{ \pm} \equiv(1 \pm \sqrt{1-\tau})
$$

Dentre as contribuições fermiônicas, a dominante é a do quark top, pois a contribuição é proporcional à massa do férmion. Ela será a única considerada aqui com exceção de uma possível partícula genérica $f$ adicional. De (2.8) e (2.9) podemos expressar $g_{H f \bar{f}}=\frac{m_{f}}{v}$. Portanto, considerando os seguintes valores para o quark top: $N_{c t}=3, Q_{t}=+\frac{2}{3}$ e $\tau_{t}=\frac{4 m_{t}^{2}}{m_{H}^{2}}$ em $(2.15)$, temos a seguinte contribuição desta partícula:

$$
\mathcal{M}_{t}=-i \frac{\alpha m_{H}^{2}}{4 \pi} \frac{8}{3 v}\left(-2 \tau_{t}\left[1+\left(1-\tau_{t}\right) f\left(\tau_{t}\right)\right]\right)
$$

\subsection{A contribuição dos loops dos bósons de gauge $\mathbf{W}$ no decaimento $H \rightarrow \gamma \gamma$}

A seguir, temos os diagramas de Feynman da contribuição dos loops de W em ordem mais baixa da teoria de perturbações para o decaimento $H \rightarrow \gamma \gamma$ : 


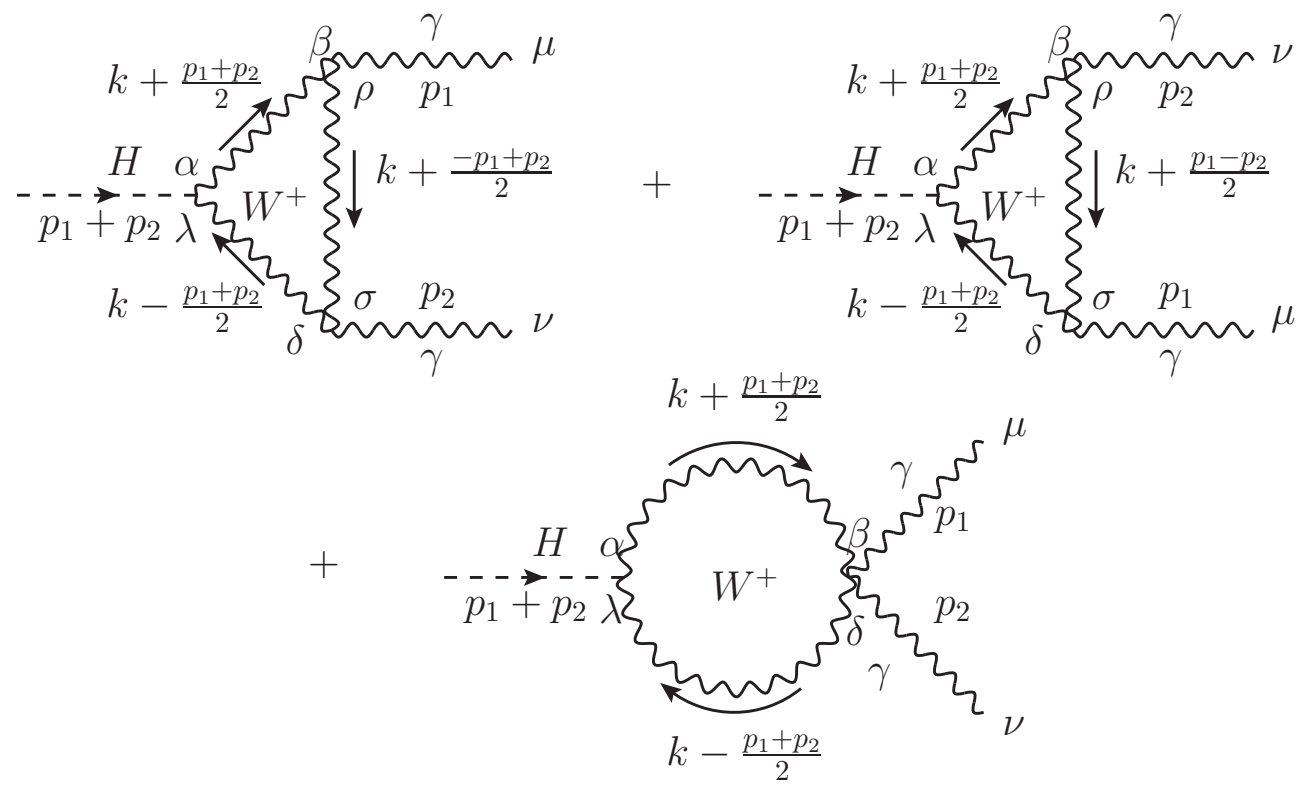

Definindo $q_{1} \equiv k+\frac{p_{1}+p_{2}}{2}, q_{2} \equiv k+\frac{-p_{1}+p_{2}}{2}, q_{3} \equiv k-\frac{p_{1}+p_{2}}{2}$ e obedecendo as regras de Feynman apresentadas no início do capítulo, obtemos a amplitude para o primeiro diagrama do loop de bósons $\mathrm{W}$ :

$$
\begin{aligned}
\mathcal{M}_{W 1} & =i \frac{2 m_{W}^{2}}{v} e^{2} \epsilon^{\mu *}\left(p_{1}\right) \epsilon^{\nu *}\left(p_{2}\right) \times \\
& \times \int \frac{d^{4} k}{(2 \pi)^{4}} \frac{1}{q_{1}^{2}-m_{W}^{2}}\left(-g^{\alpha \beta}+\frac{q_{1}^{\alpha} q_{1}^{\beta}}{m_{W}^{2}}\right) V_{\mu \rho \beta}\left(-p_{1},-q_{2}, q_{1}\right) \times \\
& \times \frac{1}{q_{2}^{2}-m_{W}^{2}}\left(-g^{\rho \sigma}+\frac{q_{2}^{\rho} q_{2}^{\sigma}}{m_{W}^{2}}\right) V_{\nu \delta \sigma}\left(-p_{2},-q_{3}, q_{2}\right) \times \\
& \times \frac{1}{q_{3}^{2}-m_{W}^{2}}\left(-g^{\alpha \delta}+\frac{q_{3}^{\alpha} q_{3}^{\delta}}{m_{W}^{2}}\right),
\end{aligned}
$$

onde $V_{\mu \nu \delta}\left(p_{1}, p_{2}, p_{3}\right)$ é definido nas regras de Feynman apresentadas acima.

Usando a definição de $q_{1}$ e $q_{3}$ acima e definindo $\bar{q}_{2}=k+\frac{p_{1}-p_{2}}{2}$, obtemos o segundo diagrama a partir das seguintes trocas na amplitude $\mathcal{M}_{W 1}: q_{2} \rightarrow \bar{q}_{2}$, 
$p_{1} \leftrightarrow p_{2}$ e $\mu \leftrightarrow \nu$. Portanto, temos:

$$
\begin{aligned}
\mathcal{M}_{W 3} & =i \frac{2 m_{W}^{2}}{v} e^{2} \epsilon^{\mu *}\left(p_{1}\right) \epsilon^{\nu *}\left(p_{2}\right) \times \\
& \times \int \frac{d^{4} k}{(2 \pi)^{4}} \frac{1}{q_{1}^{2}-m_{W}^{2}}\left(-g^{\alpha \beta}+\frac{q_{1}^{\alpha} q_{1}^{\beta}}{m_{W}^{2}}\right) V_{\nu \rho \beta}\left(-p_{2},-\bar{q}_{2}, q_{1}\right) \times \\
& \times \frac{1}{\bar{q}_{2}^{2}-m_{W}^{2}}\left(-g^{\rho \sigma}+\frac{\bar{q}_{2}^{\rho} \bar{q}_{2}^{\sigma}}{m_{W}^{2}}\right) V_{\mu \delta \sigma}\left(-p_{1},-q_{3}, \bar{q}_{2}\right) \times \\
& \times \frac{1}{q_{3}^{2}-m_{W}^{2}}\left(-g^{\alpha \delta}+\frac{q_{3}^{\alpha} q_{3}^{\delta}}{m_{W}^{2}}\right) .
\end{aligned}
$$

De forma semelhante à primeira amplitude, temos $q_{1} \equiv k+\frac{p_{1}+p_{2}}{2}, q_{3} \equiv$ $k-\frac{p_{1}+p_{2}}{2}$. Assim, obtemos a amplitude para o terceiro diagrama do loop de bósons W:

$$
\begin{aligned}
\mathcal{M}_{W 2} & =i \frac{2 m_{W}^{2}}{v} e^{2} \epsilon^{\mu *}\left(p_{1}\right) \epsilon^{\nu *}\left(p_{2}\right) \times \\
& \times \int \frac{d^{4} k}{(2 \pi)^{4}} \frac{1}{q_{1}^{2}-m_{W}^{2}}\left(-g^{\alpha \beta}+\frac{q_{1}^{\alpha} q_{1}^{\beta}}{m_{W}^{2}}\right) U_{\beta \delta \mu \nu} \times \\
& \times \frac{1}{q_{3}^{2}-m_{W}^{2}}\left(-g^{\delta \alpha}+\frac{q_{3}^{\delta} q_{3}^{\alpha}}{m_{W}^{2}}\right)
\end{aligned}
$$

onde $U_{\mu \nu \delta \lambda}$ é definido nas regras de Feynman apresentadas acima.

O resultado da contribuição do loop de bósons W é dado pela soma $\mathcal{M}_{W}=$ $\mathcal{M}_{W 1}+\mathcal{M}_{W 2}+\mathcal{M}_{W 3}$. O resultado da amplitude final da contribuição após a resolução das integrais de loop e a soma sobre as polarizações dos fótons finais é, portanto:

$$
\mathcal{M}_{W}=-i \frac{\alpha}{4 \pi} \frac{2 m_{H}^{2}}{v}\left(2+3 \tau_{W}+3 \tau_{W}\left(2-\tau_{W}\right) f\left(\tau_{W}\right)\right),
$$

onde $\tau_{W}=\frac{4 m_{W}^{2}}{m_{H}^{2}}, \alpha$ é a constante de estrutura fina e $f(\tau)$ é dado por (2.16).

\subsection{A contribuição dos loops de partículas es- calares no decaimento $H \rightarrow \gamma \gamma$}


Por último, temos os diagramas de Feynman da possível contribuição adicional de partículas escalares $S$ carregadas, em ordem mais baixa da teoria de perturbações para o decaimento $H \rightarrow \gamma \gamma$ :
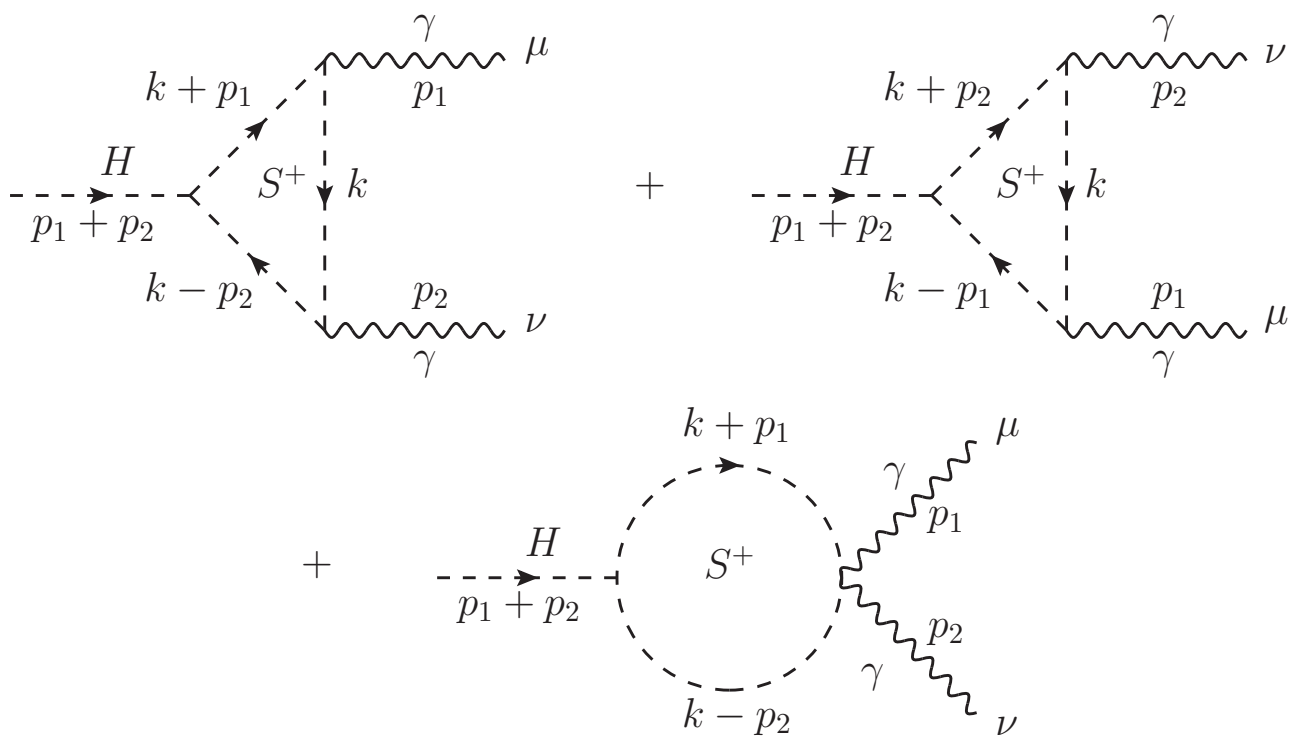

De acordo com as regras de Feynman apresentadas, temos a contribuição do primeiro diagrama do loop de partículas escalares:

$$
\begin{aligned}
\mathcal{M}_{s 1} & =-i \frac{g_{H s s} m_{H}^{2}}{2 m_{s}^{2}} e^{2} Q_{s}^{2} \epsilon_{\mu}^{*}\left(p_{1}\right) \epsilon_{\nu}^{*}\left(p_{2}\right) \times \\
& \times \int \frac{d^{4} k}{(2 \pi)^{4}} \frac{\left(2 k+p_{1}\right)^{\mu}\left(2 k-p_{2}\right)^{\nu}}{\left[\left(k+p_{1}\right)^{2}-m_{s}^{2}\right]\left[k^{2}-m_{s}^{2}\right]\left[\left(k-p_{2}\right)^{2}-m_{s}^{2}\right]} .
\end{aligned}
$$

Com as trocas $p_{1} \leftrightarrow p_{2}$ e $\mu \leftrightarrow \nu$ na amplitude (2.23), obtemos a segunda aplitude $\mathcal{M}_{s 3}$ :

$$
\begin{aligned}
\mathcal{M}_{s 3} & =-i \frac{g_{H s s} m_{H}^{2}}{2 m_{s}^{2}} e^{2} Q_{s}^{2} \epsilon_{\mu}^{*}\left(p_{1}\right) \epsilon_{\nu}^{*}\left(p_{2}\right) \times \\
& \times \int \frac{d^{4} k}{(2 \pi)^{4}} \frac{\left(2 k+p_{2}\right)^{\nu}\left(2 k-p_{1}\right)^{\mu}}{\left[\left(k+p_{2}\right)^{2}-m_{s}^{2}\right]\left[k^{2}-m_{s}^{2}\right]\left[\left(k-p_{1}\right)^{2}-m_{s}^{2}\right]} .
\end{aligned}
$$

Analogamente a $\mathcal{M}_{s 1}$ e $\mathcal{M}_{s 3}$, obtemos a amplitude para o terceiro dia- 
grama do loop de escalares pelas regras de Feynman:

$$
\begin{aligned}
\mathcal{M}_{s 2} & =i \frac{g_{H s s} m_{H}^{2}}{m_{s}^{2}} e^{2} Q_{s}^{2} g^{\mu \nu} \epsilon_{\mu}^{*}\left(p_{1}\right) \epsilon_{\nu}^{*}\left(p_{2}\right) \times \\
& \times \int \frac{d^{4} k}{(2 \pi)^{4}} \frac{1}{\left[\left(k+p_{1}\right)^{2}-m_{s}^{2}\right]\left[\left(k-p_{2}\right)^{2}-m_{s}^{2}\right]} .
\end{aligned}
$$

Por fim, o resultado desta contribuição é dado pela soma $\mathcal{M}_{s}=\mathcal{M}_{s 1}+$ $\mathcal{M}_{s 2}+\mathcal{M}_{s 3}$. O resultado final desta amplitude após a resolução das integrais de loop e a soma sobre as polarizações dos fótons finais é, portanto:

$$
\mathcal{M}_{s}=-i \frac{\alpha m_{H}^{2}}{4 \pi} \frac{g_{H s s}}{m_{s}^{2}} \tau_{s}\left[1-\tau_{s} f\left(\tau_{s}\right)\right]
$$

onde $\tau_{s}=\frac{4 m_{s}^{2}}{m_{H}^{2}}$, $\alpha$ é a constante de estrutura fina, $f(\tau)$ é dado por (2.16). Estamos considerando escalares de carga $+e$ e sem cor que aparecem em extensões do MP como modelos com dois dubletos de Higgs.

\subsection{A largura do bóson de Higgs no decai- mento $H \rightarrow \gamma \gamma$}

Nesta seção, iremos utilizar os resultados apresentados acima para obter uma expressão final da largura $\Gamma(H \rightarrow \gamma \gamma)$ deixando livre o acoplamento $g_{\text {Hss. }}$ Usamos os resultados $(2.15),(2.22)$ e (2.26) em (2.7) para obter a largura de decaimento do bóson de Higgs em dois fótons em um modelo composto por um conjunto de partículas de spin $0,1 / 2$ e 1 . Desta forma apresentada, a largura pode ser expressa como:

$$
\Gamma(H \rightarrow \gamma \gamma)=\frac{\alpha^{2} g^{2}}{1024 \pi^{3}} \frac{m_{H}^{3}}{m_{W}^{2}}\left|\sum_{f} N_{c f} Q_{f}^{2} F_{1 / 2}+F_{1}+\frac{g_{H s s}}{g} \frac{m_{W}}{m_{H}^{2}} F_{0}\right|^{2},
$$

onde as funções $F_{i}(\tau)$ são dadas por:

$$
\begin{aligned}
F_{1 / 2}(\tau) & =-2 \tau[1+(1-\tau) f(\tau)], \\
F_{1}(\tau) & =2+3 \tau+3 \tau(2-\tau) f(\tau) \\
F_{0}(\tau) & =\tau[1-\tau f(\tau)],
\end{aligned}
$$


e o índice $i$ refere-se ao spin da partícula e a função $f(\tau)$ é dada por (2.16). Para as partículas do MP o acoplamento com o bóson de Higgs é dado em termos de $g$, como mostrado em (2.8) e (2.9). $Q_{f}$ é a carga elétrica da partícula $f$ dada em unidades da carga $e, N_{c f}$ é o número de cores da partícula $f$ e $\tau$ é dado por:

$$
\tau=\frac{4 m_{f}^{2}}{m_{H}^{2}}
$$

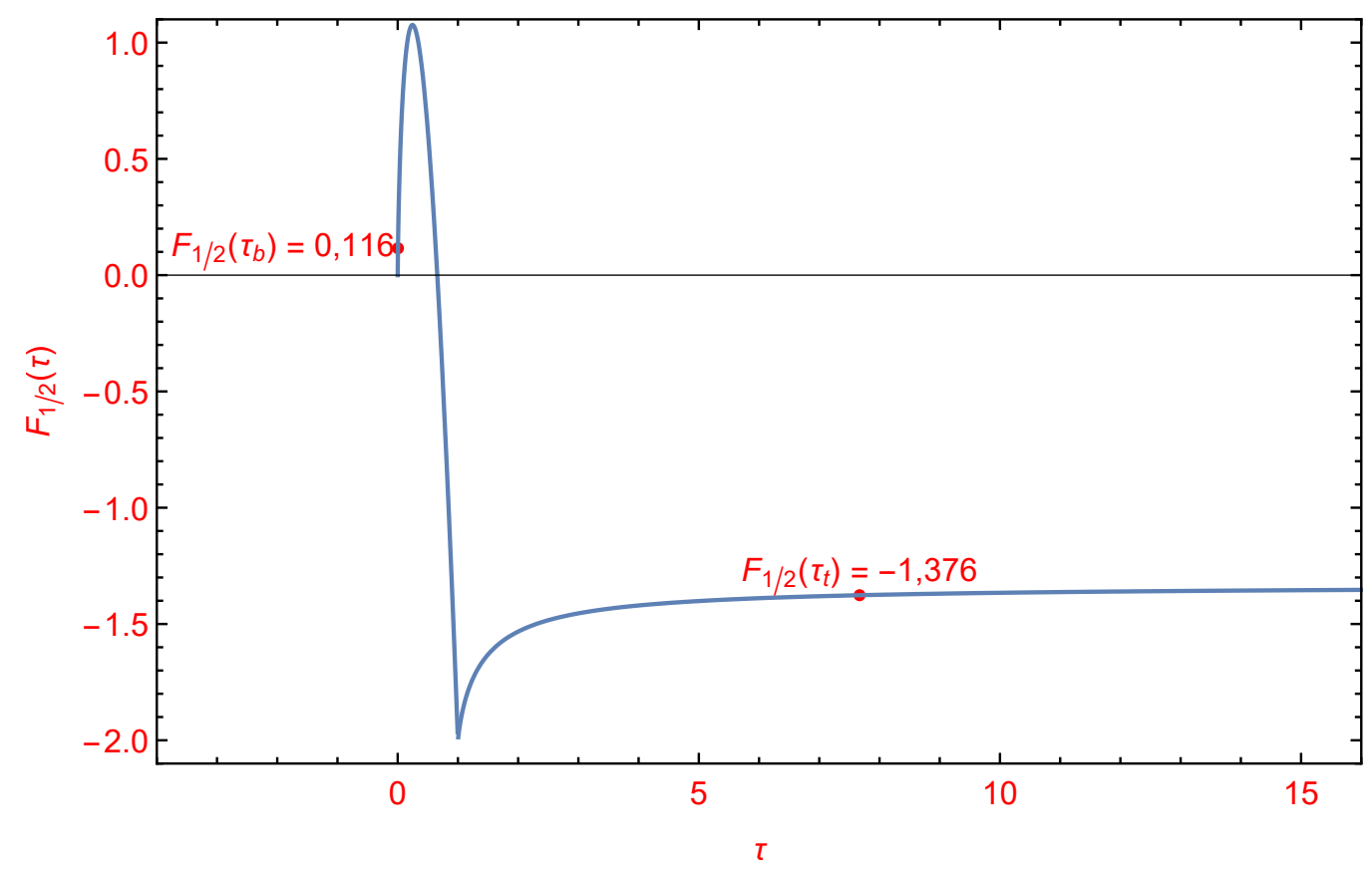

Figura 2.1: Função de loop para contribuição das partículas de spin $\frac{1}{2}$, equação (2.28).

As funções de loop para cada tipo de partícula estão representadas pelos gráficos das Figuras 2.1, 2.2 e 2.3. Na figura 2.1, dois pontos destacam os valores de $F_{1 / 2}(\tau)$ para o quark top e o quark bottom. Os valores de $\tau$ para cada tipo de partícula foram calculados considerando $m_{t}=173,21 \mathrm{GeV}$, $m_{b}=4,18 \mathrm{GeV}$ e $m_{H}=125,09 \mathrm{GeV}$ [49]. Na Figura 2.2 está indicado o $F_{1}(\tau)$ para o bóson de gauge $\mathrm{W}$, cujo valor foi obtido para $m_{W}=80,385$ $\mathrm{GeV}$ e $m_{H}=125,09 \mathrm{GeV}$ [49]. 


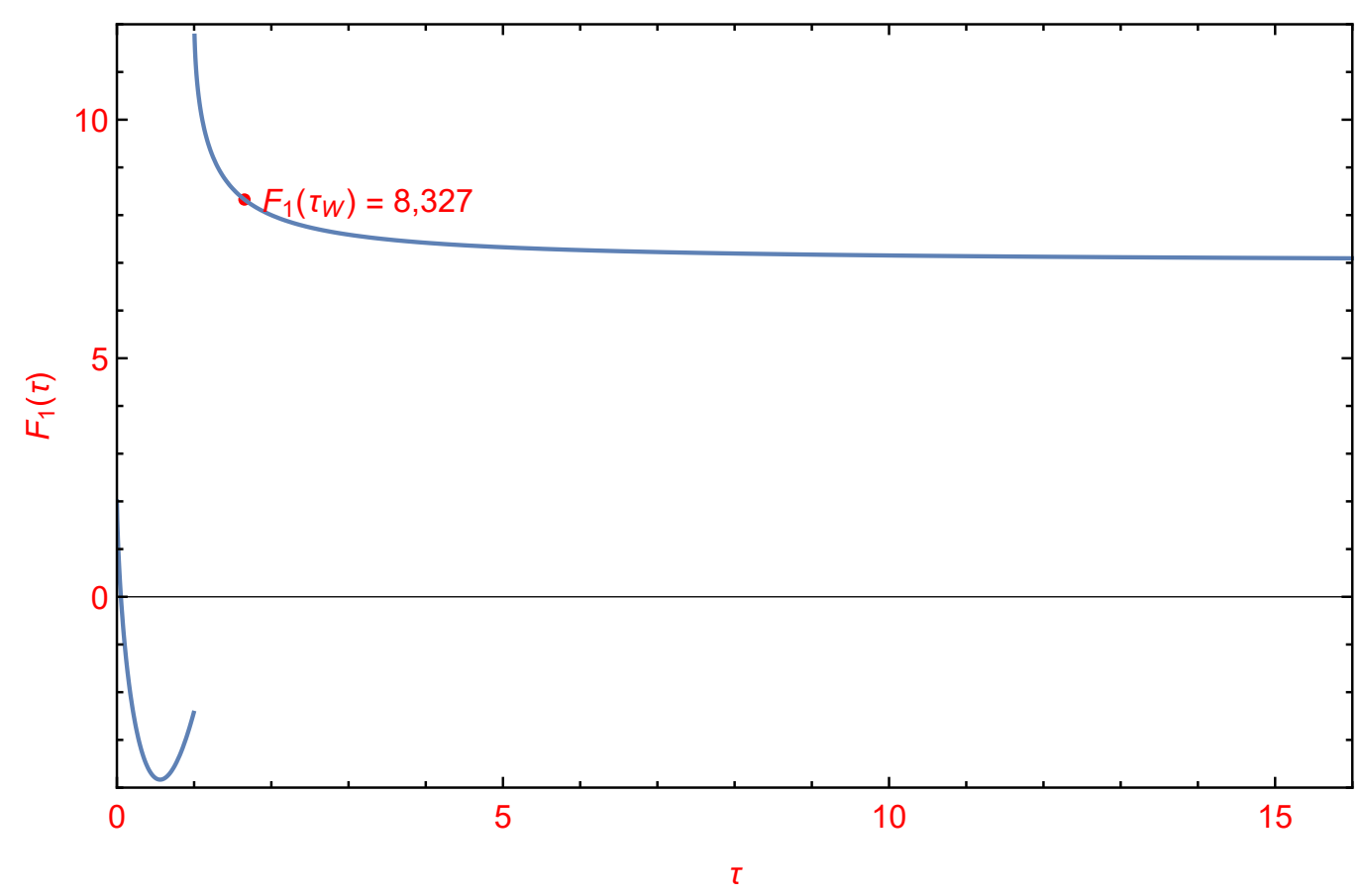

Figura 2.2: Função de loop para contribuição das partículas de spin 1, equação (2.29).

É notável, a partir da equação (2.27), que a largura de decaimento é sensível ao valor das funções de loop. Percebe-se nas Figuras 2.1 e 2.2 que as funções de loop do quark top e do bóson $W$ são dominantes. Na equação (2.27), estas partículas contribuem com

$$
\begin{array}{rc}
\text { bóson } W \rightarrow & F_{1}\left(\tau_{W}\right)=8,327, \\
\text { quark top } \rightarrow & 3 \times(2 / 3)^{2} \times F_{1 / 2}\left(\tau_{t}\right)=-1,835 \mathrm{e} \\
\text { quark bottom } \rightarrow & 3 \times(1 / 3)^{2} \times F_{1 / 2}\left(\tau_{b}\right)=0,039
\end{array}
$$

no somatório. As contribuições do restante das partículas fermiônicas se tornam cada vez mais desprezíveis à medida que suas massas diminuem, como pode ser visto pelo comportamento da função de loop para $\tau<\tau_{b}$ na Figura 2.1. Vemos que, para $m_{f} \leq m_{b}$, essas contribuições são positivas somando um total de 0,096 incluindo todos os férmions do MP com massa menor que a do quark top. Como o top e o bottom são as duas partículas fermiônicas com 


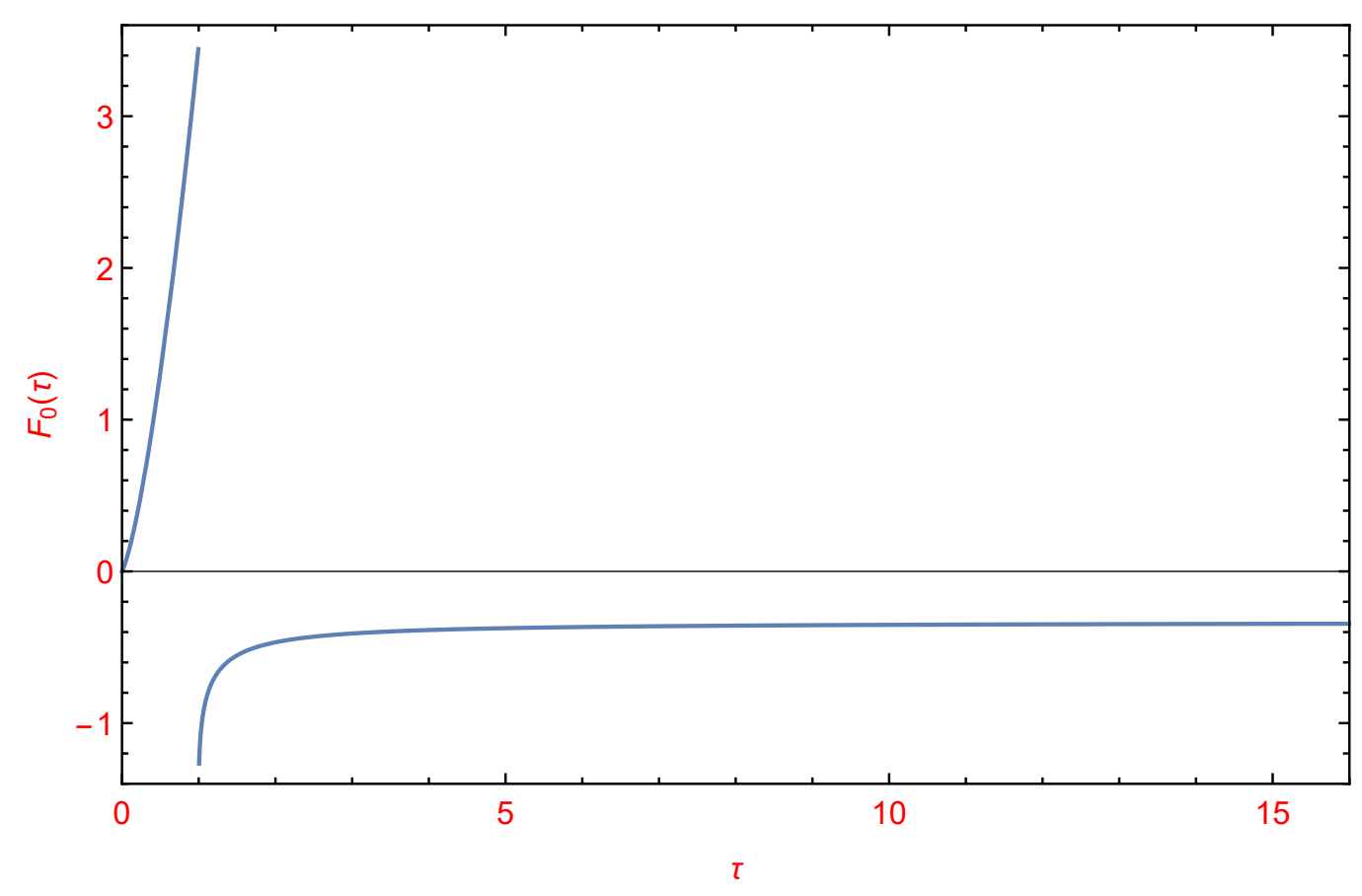

Figura 2.3: Função de loop para contribuição das partículas de spin 0, equação $(2.30)$.

maior massa conhecida, então pode-se desprezar quaisquer férmions que não seja o quark top no cálculo de $\Gamma(H \rightarrow \gamma \gamma)$ e incluir as outras contribuições na incerteza teórica da estimativa.

Observando as Figuras 2.1 e 2.3, é possível ver como a adição de partículas poderia afetar a largura. Valores positivos ou negativos dessas funções de loop poderia contribuir para o aumento ou diminuição do valor do $\Gamma(H \rightarrow \gamma \gamma)$.

No MP, apenas as contribuições do quark top e dos bósons $W$ são consideradas. Portanto, conhecendo os valores do valor esperado de vácuo, $v$, da massa do bóson de Higgs, $m_{H}$, e das funções de loop para cada uma dessas partículas é possível obter um valor para a largura de decaimento de $H \rightarrow \gamma \gamma$ previsto pelo MP. Como $v \approx 246 \mathrm{GeV}, F_{1 / 2}\left(\tau_{t}\right)=-1,376$ e $F_{1}\left(\tau_{W}\right)=8,327$ então a largura para $m_{H}=125,09 \mathrm{GeV}$ é dada por:

$$
\Gamma_{M P}(H \rightarrow \gamma \gamma)=(9,165 \pm 0,134) \mathrm{keV},
$$

onde estimamos a incerteza devido aos demais férmions do MP. 
O valor previsto pelo MP para a largura total do bóson de Higgs considerando uma massa de $m_{H}=125,09 \mathrm{GeV}$ é de $\Gamma_{H}=(4,100 \pm 0,030)$ $\mathrm{MeV}$ [50]. Desta forma, obtemos a razão de ramificação para o decaimento $H \rightarrow \gamma \gamma$ usando (2.35):

$$
\frac{\Gamma_{M P}(H \rightarrow \gamma \gamma)}{\Gamma_{H}}=2,24 \cdot 10^{-3}
$$

O que é perfeitamente compatível com $(2,28 \pm 0,11) \cdot 10^{-3}[50]$.

Podemos agora nos perguntar se ainda é possível adicionar um escalar. Nesse caso, a amplitude total do decaimento é dada como $\mathcal{M}=\mathcal{M}_{t}+\mathcal{M}_{W}+$ $\mathcal{M}_{s}$. Assim, a largura (2.7) do processo para este modelo, considerando $g_{H s s}$ como um parâmetro livre, é:

$$
\Gamma(H \rightarrow \gamma \gamma)=\frac{\alpha^{2} m_{H}^{3}}{1024 \pi^{3}}\left|\frac{8}{3 v} F_{1 / 2}\left(\tau_{t}\right)+\frac{2}{v} F_{1}\left(\tau_{W}\right)+\frac{g_{H s s}}{m^{2}} F_{0}\left(\tau_{s}\right)\right|^{2}
$$

Para $0 \leq g_{H s s} \leq 4 \pi$ observamos que a contribuição de um escalar carregado pode no máximo aumentar $\Gamma_{M P}(H \rightarrow \gamma \gamma)$ de $9,165 \mathrm{keV}$ para 9,192 $\mathrm{keV}$, o que é compatível com a incerteza da largura. Concluímos que um escalar carregado, que contribui para $H \rightarrow \gamma \gamma$ ainda é permitido. 


\section{Conclusões}

A largura do bóson de Higgs no decaimento em dois fótons é calculada por meio das regras de Feynman obtidas das interações entre campos do MP com a incorporação do mecanismo de Higgs. Uma expressão para a largura é obtida calculando as contribuições de 1-loop de férmions elementares e bósons de gauge W. Estas partículas são as únicas que se acoplam tanto com o bóson de Higgs quanto com fótons de forma mais significativa, de acordo com as previsões do MP.

Em modelos além do MP consideramos haver contribuições de novas partículas. De forma geral, é possível adicionar contribuições de loops formados por novos férmions ou escalares. A expressão para a largura de decaimento fica, portanto, em termos dos acoplamentos e massas destas novas partículas. Comentamos como a parametrização de Feynman e a regularização dimensional são necessárias como ferramentas de manipulação para resolver cálculos de integrais de loop e eliminar as divergências logarítmicas.

Foi obtida uma expressão geral para a largura $\Gamma(H \rightarrow \gamma \gamma)$ a partir da amplitude $\mathcal{M}$ composta pela a união das contribuições de $W$, férmions e escalares: (2.15), (2.22), (2.26), respectivamente. Esta largura é expressa por (2.27) e pelas funções de loop (2.28), (2.29) e (2.30).

As Figuras 2.1, 2.2 e 2.3 representam funções de loop e servem para ilustrar o quão sensível estas funções são em relação às massas das partículas. Os dois primeiros gráficos esclarecem como o quark top e o bóson $W$ dominam a largura do decaimento. As contribuições delas somadas representam quase 
duzentas vezes as contribuições das outras partículas, justificando o desprezo de quaisquer férmions que não seja o quark top no cálculo de $\Gamma(H \rightarrow \gamma \gamma)$.

Em posse dos valores dos acoplamentos e das imagens das funções de loop para o bóson $W$ e quark top, foi estimado o valor $(9,165 \pm 0,134) \mathrm{keV}$ para $\Gamma(H \rightarrow \gamma \gamma)$. Este valor considera apenas as duas contribuições dominates. Incluímos na incerteza da previsão da largura o efeito dos demais férmions. Utilizando o valor previsto para a largura total do bóson de Higgs, foi obtido $2,24 \cdot 10^{-3}$ para a razão de ramificação para o canal $H \rightarrow \gamma \gamma$.

Em particular, calculamos a largura do processo com a adição de um escalar dado por (2.37). Observa-se que a largura de decaimento é sensível ao valor das funções de loop. Isto torna possível alterar a largura a partir desta adição de partículas. Contribuições negativas e positivas podem contribuir para aumento ou diminuição do valor da largura do decaimento, como se vê nas Figuras 2.1 e 2.3. No entanto, para $m_{s}>100 \mathrm{GeV}$ e $0 \leq g_{H s s} \leq$ $4 \pi$, é possível obter uma largura $H \rightarrow \gamma \gamma$ com valores dentro da incerteza experimental. Concluímos quer a largura de $H$ em dois $\gamma$ 's ainda permite a existência de um bóson escalar carregado que se acopla ao Higgs. 


\section{Apêndice A}

\section{Técnicas para cálculos de integrais de loop}

\section{A.1 Propriedades do traço das matrizes $\gamma$}

Em cálculos envolvendo loops de partículas fermiônicas é comum surgir traços de matrizes $\gamma$ no numerador do integrando. Aqui, ele aprece na contribuição fermiônica do decaimento $H \rightarrow \gamma \gamma$ mostrado na seção 2.1. As seguintes propriedades são úteis para a resolução do problema:

$$
\begin{aligned}
\operatorname{tr}[1] & =4 \\
\operatorname{tr}\left[\text { número ímpar de } \gamma^{\prime} s\right] & =0 \\
\operatorname{tr}\left[\gamma^{\mu} \gamma^{\nu}\right] & =4 g^{\mu \nu} \\
\operatorname{tr}\left[\gamma^{\mu} \gamma^{\nu} \gamma^{\rho} \gamma^{\sigma}\right] & =4\left(g^{\mu \nu} g^{\rho \sigma}-g^{\mu \rho} g^{\nu \sigma}+g^{\mu \sigma} g^{\nu \rho}\right) \\
\operatorname{tr}\left[\gamma^{5}\right] & =0 \\
\operatorname{tr}\left[\gamma^{\mu} \gamma^{\nu} \gamma^{5}\right] & =0 \\
\operatorname{tr}\left[\gamma^{\mu} \gamma^{\nu} \gamma^{\rho} \gamma^{\sigma} \gamma^{5}\right] & =-4 i \epsilon^{\mu \nu \rho \sigma}
\end{aligned}
$$




\section{A.2 Parâmetros de Feynman}

Esta técnica é útil para simplificar expressões para propagadores internos de loops com a finalidade de aplicar a regularização dimensional. A partir da adição de parâmetros reais conhecidos como parâmetros de Feynman, ela exibe frações usuais como integrais. A seguir, exibimos as frações mais apropriadas para os problemas abordados neste trabalho:

$$
\begin{gathered}
\frac{1}{a b}=\int_{0}^{1} d x \frac{1}{[(1-x) b+x a]^{2}}, \\
\frac{1}{a b c}=2 \int_{0}^{1} d x \int_{0}^{1} d y \frac{x}{[a x y+b x(1-y)+c(1-x)]^{3}}= \\
=2 \int_{0}^{1} d u \int_{0}^{1-u} d w \frac{1}{[a w+b(1-u-w)+c u]^{3}} .
\end{gathered}
$$

Como exemplo, vamos representar a expressão de (2.10)

$$
\frac{1}{\left[\left(k+p_{1}\right)^{2}-m_{f}^{2}\right]\left[k^{2}-m_{f}^{2}\right]\left[\left(k-p_{2}\right)^{2}-m_{f}^{2}\right]}
$$

utilizando parâmetros de Feynman. Para começar, definimos $a \equiv\left(k+p_{1}\right)^{2}-$ $m_{f}^{2}, b \equiv k^{2}-m_{f}^{2}$ e $c \equiv\left(k-p_{2}\right)^{2}-m_{f}^{2}$, e substituímos estes termos na segunda igualdade de (A.9):

$$
\begin{aligned}
& \frac{1}{a b c}=2 \int_{0}^{1} d u \int_{0}^{1-u} d w \\
& \frac{1}{\left[\left(\left(k+p_{1}\right)^{2}-m_{f}^{2}\right) w+\left(k^{2}-m_{f}^{2}\right)(1-u-w)+\left(\left(k-p_{2}\right)^{2}-m_{f}^{2}\right) u\right]^{3}}=(\mathrm{A} .11) \\
& =2 \int_{0}^{1} d u \int_{0}^{1-u} d w \frac{1}{\left[k^{2}+2 k \cdot\left(w p_{1}-u p_{2}\right)-m_{f}^{2}\right]^{3}} .
\end{aligned}
$$

É de bastante utilidade completar o quadrado na variável $k$ de $k^{2}+2 k \cdot\left(w p_{1}-\right.$ $\left.u p_{2}\right)-m_{f}^{2}$ do denominador. Desta forma, é possível expressar o resultado acima como:

$$
\frac{1}{a b c}=2 \int_{0}^{1} d u \int_{0}^{1-u} d w \frac{1}{\left[l^{2}-\Lambda\right]^{3}},
$$


onde $l$ é dado em função de $k, w, p_{1}, p_{2}$ e $m_{f}$; e $\Lambda$ em função das mesmas variáveis com exceção de $k$.

Este procedimento é feito analogamente a este exemplo em todas as integrais de loop envolvidas no decaimento $H \rightarrow \gamma \gamma$. Em loops com dois propagadores internos, como os encontrados nas contribuições de loop de $W$ e de escalar, usa-se a relação (A.8) e em loops com três propagadores internos usa-se (A.9).

\section{A.3 Regularização dimensional}

As integrais de loop, quando formulada em termos dos parâmetros de Feynman, permite-nos usar a regularização dimensional na variável $l$ ao inverter a ordem de integração. Como exemplo, o resultado da parametrizacão (A.12) é aplicado em (2.10). Uma soma de termos proporcionais a (2.12), (2.13) e (2.14) é obtida.

As seguintes soluções servem para esse tipo de problema e demais cálculos de integrais loop:

$$
\begin{gathered}
\int \frac{d^{d} l}{(2 \pi)^{d}} \frac{1}{\left[l^{2}-\Lambda\right]^{n}}=\frac{(-1)^{n} i}{(4 \pi)^{\frac{d}{2}}} \frac{\Gamma\left(n-\frac{d}{2}\right)}{\Gamma(n)}\left(\frac{1}{\Lambda}\right)^{n-\frac{d}{2}} \\
\int \frac{d^{d} l}{(2 \pi)^{d}} \frac{l^{2}}{\left[l^{2}-\Lambda\right]^{n}}=\frac{(-1)^{n-1} i}{(4 \pi)^{\frac{d}{2}}} \frac{d}{2} \frac{\Gamma\left(n-\frac{d}{2}-1\right)}{\Gamma(n)}\left(\frac{1}{\Lambda}\right)^{n-\frac{d}{2}-1}, \\
\int \frac{d^{d} l}{(2 \pi)^{d}} \frac{l^{\mu} l^{\nu}}{\left[l^{2}-\Lambda\right]^{n}}=\frac{(-1)^{n-1} i}{(4 \pi)^{\frac{d}{2}}} \frac{g^{\mu \nu}}{2} \frac{\Gamma\left(n-\frac{d}{2}-1\right)}{\Gamma(n)}\left(\frac{1}{\Lambda}\right)^{n-\frac{d}{2}-1}, \\
\int \frac{d^{d} l}{(2 \pi)^{d}} \frac{\left(l^{2}\right)^{2}}{\left[l^{2}-\Lambda\right]^{n}}=\frac{(-1)^{n} i \frac{d(d+2)}{(4 \pi)^{\frac{d}{2}}} \frac{\Gamma\left(n-\frac{d}{2}-1\right)}{\Gamma(n)}\left(\frac{1}{\Lambda}\right)^{n-\frac{d}{2}-2} \mathrm{e}}{\int \frac{d^{d} l}{(2 \pi)^{d}} \frac{l^{\mu} l^{\nu} l^{\rho} l^{\sigma}}{\left[l^{2}-\Lambda\right]^{n}}=\frac{(-1)^{n} i}{(4 \pi)^{\frac{d}{2}}} \frac{1}{4} \frac{\Gamma\left(n-\frac{d}{2}-2\right)}{\Gamma(n)}\left(\frac{1}{\Lambda}\right)^{n-\frac{d}{2}-2} \times} \\
\times\left(g^{\mu \nu} g^{\rho \sigma}+g^{\mu \rho} g^{\nu \sigma}+g^{\mu \sigma} g^{\nu \rho}\right) .
\end{gathered}
$$


Algumas destas integrais apresentam divergências nos cálculos referentes a este trabalho. Nos casos onde temos $n=2$ e $d=4$ em (A.13), $n=3$ e $d=4$ em (A.14) e (A.15) a função $\Gamma$ apresenta argumento zero, portanto, é uma função divergente. Nestes casos, desconsideramos $d=4$ no princípio e expressamos

$$
\Gamma\left(2-\frac{d}{2}\right) \text { e }\left(\frac{1}{\Lambda}\right)^{2-\frac{d}{2}}
$$

como

$$
\Gamma\left(\frac{\epsilon}{2}\right) \text { e }\left(\frac{1}{\Lambda}\right)^{\frac{\epsilon}{2}}
$$

para valores de $\epsilon$ tendendo a zero, onde $\epsilon=4-d$. Desta forma, temos:

$$
\frac{\Gamma\left(\frac{\epsilon}{2}\right)}{(4 \pi)^{\frac{d}{2}}}\left(\frac{1}{\Lambda}\right)^{\frac{\epsilon}{2}}=\frac{1}{(4 \pi)^{\frac{d}{2}}}(\delta \epsilon-\log \Lambda),
$$

onde $\delta \epsilon=\frac{2}{\epsilon}-\gamma+\mathcal{O}(\epsilon)$ é um termo que seria divergente para $d=4$ e é eliminado por cancelamento simples na computação da largura $\Gamma(H \rightarrow \gamma \gamma)$. 


\section{Referências Bibliográficas}

[1] S. L. Glashow, "Partial-symmetries of weak interactions", Nucl. Phys. 22 no. 4, (1961) 579.

[2] S. Weinberg, "A Model of Leptons", Phys. Rev. Lett. 19 (1967) 1264.

[3] A. Salam, "Weak and electromagnetic interactions", em Elementary particle theory: relativistic groups and analyticity, N. Svartholm, ed., p. 367. Almqvist \& Wiksell, 1968. Proceedings of the eighth Nobel symposium.

[4] G. 'т Hooft; M. Veltman, "Regularization and Renormalization of Gauge Fields", Nucl. Phys. B44 (1972) 189.

[5] Englert, F.; Brout, R. Phys. Rev. Lett. 13, 321 (1964).

[6] Higgs, P. W., "Broken symmetries, massless particles and gauge fields", Phys. Lett. 12 (1964) 132.

[7] Higgs, P. W. Phys. Rev. Lett. 13, 508 (1964).

[8] Guralnik, G. S.; Hagen, C. R.; Kibble, T. W. B. Phys. Rev. Lett. 13, 585 (1964).

[9] Higgs, P. W., "Spontaneous symmetry breakdown without massless bosons", Phys. Rev. 145 (1966) 1156.

[10] KiBBle, T. W. B., "Symmetry breaking in non-Abelian gauge theories", Phys. Rev. 155 (1967) 1554. 
[11] ATLAS Collaboration, G. Aad et al., "Observation of a new particle in the search for the Standard Model Higgs boson with the ATLAS detector at the LHC', arXiv:1207.7214 [hep-ex].

[12] CMS Collaboration, S. Chatrchyan et al., "Observation of a new boson at a mass of $125 \mathrm{GeV}$ with the CMS experiment at the $\mathrm{LHC}^{\circ}$, arXiv:1207.7235 [hep-ex].

[13] L. Evans e P. Bryant (editores), "LHC Machine", JINST 3 (2008) S08001, doi:10.1088/1748-0221/3/08/S08001.

[14] Nambu, Y., Phys. Rev. Letters 4, 380 (1960).

[15] Nambu, Y.; Jona-Lasinio, G., Phys. Rev. 122 (1961) 345.

[16] Baker, M.; Glashow, S. L., Phys. Rev. 128, (1962) 2462.

[17] Glashow, S. L., Phys. Rev. 130, (1962) 2132.

[18] Nambu, Y.; Pascual, P., Nuovo Cimento 30, (1963) 354.

[19] Goldstone, J., Nuovo Cimento 19 (1961) 154.

[20] Goldstone, J.; Salam, A.; Weinberg, S. Phys. Rev. 127 (1962) 965.

[21] Bludman, S. A.; Klein, A., Phys. Rev. 131 (1963) 2364.

[22] ATLAS Collaboration, "ATLAS: letter of intent for a generalpurpose pp experiment at the large hadron collider at CERN", CERNLHCC-92-004 (1992).

[23] ATLAS Collaboration, "ATLAS Technical Proposal for a GeneralPurpose pp Experiment at the Large Hadron Collider at CERN", CERNLHCC-94-43 (1994).

[24] ATLAS Collaboration, "The ATLAS Experiment at the CERN Large Hadron Collider", JINST 3 (2008) S08003. 
[25] ALEPH, CDF, DØ, DELPHI, L3, OPAL, SLD Collaborations, the LEP Electroweak Working Group, the Tevatron Electroweak Working Group, and the SLD electroweak and heavy flavour groups, "Precision Electroweak Measurements and Constraints on the Standard Model', CERN-PH-EP-2010-095 (2010), arXiv:1012.2367 [hep-ex].

[26] ALEPH, CDF, D0, DELPHI, L3, OPAL, SLD Collaborations, the LEP Electroweak Working Group, the Tevatron ElectroweakWorking Group, and the SLD Electroweak and Heavy Flavour Groups, "Precision Electroweak Measurements and Constraints on the Standard Model', CERN PH-EP-2010-095, (2010).

[27] J. M. Cornwall, D. N. Levin, and G. Tiktopoulos, "Uniqueness of spontaneously broken gauge theories", Phys. Rev. Lett. 30 (1973) 1268, doi:10.1103/PhysRevLett.30.1268.

[28] J. M. Cornwall, D. N. Levin, and G. Tiktopoulos, "Derivation of Gauge Invariance from High-Energy Unitarity Bounds on the s Matrix", Phys. Rev. D 10 (1974) 1145, doi:10.1103/PhysRevD.10.1145. Also Erratum, doi:10.1103/PhysRevD.11.972.

[29] C. H. Llewellyn Smith, "High-Energy Behavior and Gauge Symmetry", Phys. Lett. B 46 (1973) 233, doi:10.1016/0370-2693(73)90692-8.

[30] B.W. Lee, C. Quigg, and H. B. Thacker, "Weak interactions at very high energies: The role of the Higgs-boson mass", Phys. Rev. D 16 (1977) 1519, doi:10.1103/PhysRevD.16.1519.

[31] ALEPH, DELPHI, L3 and OPAL Collaborations, The LEP Working Group for Higgs boson searches, "Search for the standard model Higgs boson at LEP', Phys. Lett. B 565 (2003) 61.

[32] CDF and D0 Collaborations, "Combination of Tevatron Searches for the Standard Model Higgs Boson in the 
$W^{+} W^{-}$Decay Mode", Phys. Rev. Lett. 104 (2010) 061802, doi:10.1103/PhysRevLett.104.061802.

[33] CDF Collaboration, T. Aaltonen et al., "Combined search for the standard model Higgs boson decaying to a b $\bar{b}$ pair using the full CDF data set", accepted by Phys. Rev. Lett. (2012), arXiv:1207.1707 [hep-ex] .

[34] CDF Collaboration, DØ Collaboration, "Evidence for a particle produced in association with weak bosons and decaying to a bottomantibottom quark pair in Higgs boson searches at the Tevatron", submitted to Phys. Rev. Lett. (2012), arXiv:1207.6436 [hep-ex].

[35] Do Collaboration, V. M. Abazov et al., "Combined search for the stan-

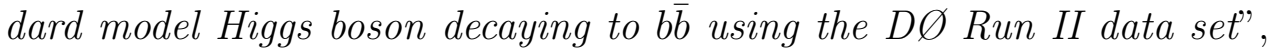
submitted to Phys. Rev. Lett. (2012), arXiv:1207.6631 [hep-ex].

[36] ATLAS Collaboration, G. Aad et al., "Search for the Standard Model Higgs boson in the diphoton decay channel with $4.9 \mathrm{fb}^{-1}$ of pp collisions at $\sqrt{s}=7 \mathrm{TeV}$ with ATLAS", arXiv:1202.1414 [hep-ex].

[37] ATLAS Collaboration, "Search for the Standard Model Higgs boson in the $H \rightarrow W W^{(*)} \rightarrow l \nu l \nu$ decay mode with $4.7 \mathrm{fb}^{-1}$ of ATLAS data at $\sqrt{s}=7$ TeV', Phys. Lett. B 716 (2012) 62.

[38] ATLAS Collaboration, "Combined search for the Standard Model Higgs boson in pp collisions at $\sqrt{s}=7$ TeV with the ATLAS detector", Phys. Rev. D86 (2012) 032003.

[39] CMS Collaboration, S. Chatrchyan et al., "Combined results of searches for the standard model Higgs boson in pp collisions at $\sqrt{s}=7$ TeV', arXiv:1202.1488 [hep-ex].

[40] M. Della Negra et al., "Muon trigger and identification", in Proceedings of the Large Hadron Collider Workshop, G. Jarlskog and D. Rein, eds., p. 467. Aachen, Germany, 1990. CERN 90-10-V-3/ECFA 90-133-V-3. 
[41] CMS Collaboration, "Letter of intent by the CMS Collaboration for a general purpose detector at the $L H C^{\prime}$, Technical Report CERN-LHCC-92-03, CERN-LHCC-I-1, CERN, (1992).

[42] N. Ellis and T. S. Virdee, "Experimental challenges in high luminosity collider physics", Ann. Rev. Nucl. Part. Sci. 44 (1994) 609, doi:10.1146/annurev.ns.44.120194.003141.

[43] CMS Collaboration, "The CMS experiment at the CERN LHC", 3 (2008) S08004, doi:10.1088/1748-0221/3/08/S08004.

[44] SeEz, C. J. ET AL., "Photon decay modes of the intermediate mass Higgs", in Proceedings of the Large Hadron Collider Workshop, G. Jarlskog and D. Rein, eds., p. 474. Aachen, Germany, 1990. CERN 90-10-V-2/ECFA 90-133-V-2.

[45] ATLAS and CMS Collaboration, "Measurements of the Higgs boson production and decay rates and constraints on its couplings from a combined ATLAS and CMS analysis of the LHC pp collision data at $\sqrt{s}=7$ TeV and 8 TeV', arXiv:1206.02266 [hep-ex].

[46] ATLAS and CMS Collaborations, Phys. Rev. Lett. 114 (2015) 191803, arXiv: 1503.07589 [hep-ex].

[47] PICH, A., "ICHEP 2014 Summary: Theory Status after the First LHC Run", arXiv:1505.01813 [hep-ph].

[48] PICH, A., "Electroweak Symmetry Breaking and the Higgs Boson", arXiv:1512.08749 [hep-ph].

[49] Olive, K. A. et al. (Particle Data Group), Chin. Phys. C, 38, 090001 (2014) and 2015 update arXiv:1512.08749 [hep-ph].

[50] LhC Higgs Cross Section Working Group, S. Heinemeier, C. Mariotti, G. Passarino, R. TAnaka (Eds.), "Handbook of LHC Higgs Cross Sections: 3. Higgs Properties", CERN?2013?004 (CERN, 
Geneva, 2013), DOI : 10.5170/CERN?2013?004.

\section{OUTROS TEXTOS:}

[51] DjouAdi, A., "The anatomy of electro-weak symmetry breaking. I: The Higgs boson in the standard model', arXiv:hep-ph/0503172.

[52] Carena, M.; Haber, H. E. "Higgs boson theory and phenomenology", Prog. Part. Nucl. Phys 50, 63 (2003), arXiv:hep-ph/0208209.

[53] Dawson, S. "Introduction to the physics of Higgs bosons", lectures given at TASI 94, arXiv:hep-ph/9411325.

[54] Dawson, S. "Introduction to electroweak symmetry breaking", lectures given at the 1998 ICTP Summer School in High-Energy Physics and Cosmology, arXiv:hep-ph/9901280.

[55] REINA, L. "TASI 2004 lecture notes on Higgs-boson physics", arXiv:hep-ph/0512377.

[56] Reina, L. "TASI 2011 lecture on Higgs-boson physics", arXiv:1208.5504v1.

[57] Peskin, M. E.; Schroeder, D. V. "An Introduction to quantum field theory", Harper Collins Publishers (June 1, 1995).

[58] Weinberg, S. "The Quantum theory of fields. Vol. 2: Modern Applications", Cambridge University Press (August 13, 1996).

[59] MatcheV, K. "TASI lectures on precision electroweak physics" arXiv:hep-ph/0402031.

[60] LEP

ElectroweakWorking

Group http: //lepewwg. web.cern.ch/LEPEWWG/.

[61] Iliopoulos, J., "Introduction to the STANDARD MODEL of the Electro-Weak Interactions", arXiv:1305.6779 [hep-ph]. 